\title{
The early inflorescence of Arabidopsis thaliana demonstrates positional effects in floral organ growth and meristem patterning
}

\author{
Andrew R. G. Plackett ${ }^{1,5}$. Stephen J. Powers ${ }^{2}$ - Andy L. Phillips ${ }^{1}$ - Zoe A. Wilson ${ }^{3}$. Peter Hedden ${ }^{1,4}$. \\ Stephen G. Thomas ${ }^{1}$
}

Received: 11 October 2017 / Accepted: 11 December 2017 / Published online: 20 December 2017

(c) The Author(s) 2017. This article is an open access publication

\begin{abstract}
Key message Linear modelling approaches detected significant gradients in organ growth and patterning across early flowers of the Arabidopsis inflorescence and uncovered evidence of new roles for gibberellin in floral development. Abstract Most flowering plants, including the genetic model Arabidopsis thaliana, produce multiple flowers in sequence from a reproductive shoot apex to form a flower spike (inflorescence). The development of individual flowers on an Arabidopsis inflorescence has typically been considered as highly stereotypical and uniform, but this assumption is contradicted by the existence of mutants with phenotypes visible in early flowers only. This phenomenon is demonstrated by mutants partially impaired in the biosynthesis of the phytohormone gibberellin (GA), in which floral organ growth is retarded in the first flowers to be produced but has recovered spontaneously by the 10th flower. We presently lack systematic data from multiple flowers across the Arabidopsis inflorescence to explain such changes. Using mutants of the GA 20-OXIDASE (GA20ox) GA biosynthesis gene family to manipulate endogenous GA levels, we investigated the dynamics of changing floral organ growth across the early Arabidopsis inflorescence (flowers 1-10). Modelling of floral organ lengths identified a significant, GA-independent gradient of increasing stamen length relative to the pistil in the wild-type inflorescence that was separable from other, GA-dependent effects. It was also found that the first flowers exhibited unstable organ patterning in contrast to later flowers and that this instability was prolonged by exogenous GA treatment. These findings indicate that the development of individual flowers is influenced by hitherto unknown factors acting across the inflorescence and also suggest novel functions for GA in floral patterning.
\end{abstract}

Keywords Arabidopsis $\cdot$ Flower $\cdot$ Inflorescence $\cdot$ Modelling $\cdot$ Gibberellin (GA)

Communicated by Dolf Weijers.

Electronic supplementary material The online version of this article (https://doi.org/10.1007/s00497-017-0320-3) contains supplementary material, which is available to authorized users.

Andrew R. G. Plackett

arp74@cam.ac.uk

1 Department of Plant Sciences, Rothamsted Research, Harpenden, Hertfordshire AL5 2JQ, UK

2 Department of Computational and Analytical Sciences, Rothamsted Research, Harpenden, Hertfordshire AL5 2JQ, UK

3 School of Biosciences, University of Nottingham, Loughborough, Leicestershire LE12 5RD, UK

4 Laboratory of Growth Regulators, Palacký University and Institute of Experimental Botany AS CR, Šlechtitelů 27, Olomouc 783 71, Czech Republic

5 Present Address: Department of Plant Sciences, University of Cambridge, Downing Street, Cambridge CB2 3EA, UK

\section{Introduction}

The development of multiple flowers from a single growth axis to form a flower spike (inflorescence) is a common characteristic of many flowering plant taxa. The model plant Arabidopsis thaliana develops an indeterminate, racemetype inflorescence (Prusinkiewicz et al. 2007), comprising individual lateral flowers arising immediately and sequentially from an apical inflorescence meristem (IM). Floral development in Arabidopsis follows a well-defined programme of events (Smyth et al. 1990) that gives rise to a stereotypical floral structure comprising a fixed sequence of concentric whorls with fixed numbers of floral organs (four sepals, four petals, six stamens and a central pistil). Organ identity within whorls is determined through overlapping expression of (and interaction between) different MADS-box genes following the ABCE model, with different whorls and 
organs within whorls separated by the expression of boundary genes (reviewed by Airoldi 2010; Irish 2010).

Despite this apparent uniformity, previous observations in Arabidopsis suggest that a flower's development is influenced by its position on the inflorescence. Mutants impaired in biosynthesis of the plant hormone gibberellin (GA) via losses of members of the GA 3-OXIDASE (GA3ox) or GA 20-OXIDASE (GA20ox) gene families demonstrate fertility and growth defects in early flowers, but not later flowers on the same plant (Hu et al. 2008; Rieu et al. 2008). Whilst GA 3-oxidase catalyses the last step in bioactive GA biosynthesis (Chiang et al. 1995; Itoh et al. 1999), in Arabidopsis GA 20-oxidase enzymes catalyse the penultimate, rate-limiting step in the GA biosynthesis pathway (Huang et al. 1998; Coles et al. 1999), and the GA20ox substrate $\mathrm{GA}_{12}$ has recently been shown to act as a long-distance growth signal in Arabidopsis (Regnault et al. 2015). Loss of three GA20ox paralogues, GA20oxl, GA20ox2 and GA20ox3, results in an infertile, GA-deficient floral phenotype in which floral organ growth is stunted (Plackett et al. 2012). In contrast, the ga20oxl ga20ox 2 mutant demonstrates a failure in siliqueset specifically in early flowers associated with reduced stamen growth relative to the pistil, but this phenotype recovers spontaneously through an unknown mechanism (Rieu et al. 2008). It has been inferred that the observed infertility in early ga20oxl ga20ox 2 flowers is caused by this mismatched growth, preventing pollination and thus silique-set. In a second mutant with a similar phenotype, ga3oxl ga3ox3, the recovery of successful silique-set in later flowers was also associated with an increase in stamen growth as flowering progressed (Hu et al. 2008). Changes in floral growth with changing position of flowers along the inflorescence have thus been demonstrated, but the mechanisms underlying those changes remain unclear.

GA itself is a candidate regulator for this process, with known roles in promoting floral organ growth and development (reviewed in Plackett et al. 2011), but the existing evidence remains inconclusive. A comparison of bioactive GA content between early (infertile) and later (fertile) ga3ox1 ga3ox3 whole floral clusters did not detect a significant difference in GA levels, suggesting that the observed phenotypic changes do not relate directly to GA (Hu et al. 2008). That said, individual, tissue-specific expression patterns have been found for each GA3ox paralogue (Mitchum et al. 2006; Hu et al. 2008) as has a complex feedback mechanism dynamically regulating the expression of different paralogues within and between the GA20ox and GA3ox gene families (Rieu et al. 2008). In floral tissues, there is differential expression between GA20ox1 and GA20ox2 in the stamen filament and pistil, respectively, and up-regulation of GA20ox3 in the ga20oxl ga20ox2 background (Plackett et al. 2012). Local changes in GA biosynthesis that affect specific organ development may thus have been missed by analyses at the whole flower level. Against this argument, spontaneous recovery of floral organ growth and silique-set has been reported during very late flowering of the GA-deficient mutant gal-3 (Cheng et al. 2004; Plackett et al. 2012), in which GA biosynthesis is entirely blocked at an early stage (Silverstone et al. 1997). However, experimental contamination by bioactive GA or the volatile GA precursor ent-kaurene (leading to endogenous GA biosynthesis in gal-3) could not be conclusively ruled out under these circumstances (King et al. 2001; Otsuka et al. 2004; Silverstone et al. 2001).

To determine the contribution of GA to changes in floral organ growth across the early inflorescence, we conducted a detailed analysis of floral phenotypes in wild type (Col-0) and all combinations of ga20oxl, ga20ox2 and ga20ox3 loss-of-function mutant alleles across the early inflorescence, taking advantage of the phenotypic variation these mutants provide: the ga20oxl single mutant shows significant reductions in silique-set across this range, but less than ga20oxl ga20ox2 (Rieu et al. 2008), whereas the ga20ox1 ga20ox2 ga20ox3 floral phenotype is similar to that of gal-3 (Plackett et al. 2012). Whilst different genotypes displayed changes to their silique-set frequency to a greater or lesser extent across the early inflorescence, our analysis identified a common trend between them of an increasing probability of silique-set with advancing flower position, irrespective of genotype or GA status. This is correlated with a significant gradient of increasing stamen length (both relative to the pistil and in absolute terms) with advancing flower position under control growth conditions in the wild-type inflorescence and most ga20ox genotypes, including ga20ox1 ga20ox2 ga20ox3. The observed reduced growth of stamens (and petals) in early, non-silique-setting flowers of ga20ox1 ga20ox2 was independent from this underlying gradient and instead correlated with a failure of anther dehiscence in these flowers. Thus, GA-dependent and GA-independent components were identified in association with changing floral organ growth and development across the early inflorescence.

Unexpectedly, we found that development in the earliest flowers to form on the Col-0 wild-type inflorescence was atypical, with significantly more aberrations in organ number and structure occurring than in later flowers. Patterning became highly stereotypical in later flowers under control growth conditions, but remained unstable under exogenous GA treatment. A genetic component was identified, with ga20oxl and ga20oxl ga20ox3 displaying altered frequencies of abnormalities. These results suggest a novel, previously unidentified role for GA in floral patterning and early floral organ development, consistent with published expression patterns for GA biosynthetic and signalling genes. 


\section{Materials and methods}

\section{Plant material and growth conditions}

The homozygous ga20ox mutant lines used in these experiments, comprising combinations of the ga20oxl-3, ga20ox2-1 and ga20ox3-1 alleles in the Col-0 background, have been previously described (Rieu et al. 2008; Plackett et al. 2012) and were compared against wild type (Col-0). Experiments characterising the frequency of silique-set during early flowering included $\mathrm{gal}-3(\mathrm{Col}-0)$ as a GAdeficient control (Tyler et al. 2004). All plants were grown on Levington F2 soil under long-day (LD) conditions (16-h light, $300 \mu \mathrm{mol} \mathrm{m} \mathrm{m}^{-2} \mathrm{~s}^{-1}, 23^{\circ} \mathrm{C} ; 8$-h darkness, $18^{\circ} \mathrm{C}$ ). Foliar treatments were applied to growing plants of each genotype three times a week from germination onwards as previously described (Rieu et al. 2008), comprising either mock treatment with water (control growth conditions) or $100 \mu \mathrm{M} \mathrm{GA}_{3}$ (GA treatment).

\section{Characterisation of silique-set across the early inflorescence}

Plants of each genotype were allowed to develop to nearmaturity, and then the presence or absence of a fertilised silique was scored across early flower positions on the primary inflorescence (unfertilised flower positions scored as ' 0 ' and successful silique-set scored as ' 1 '). The presence or absence of developing siliques was typically a binary character, with mature siliques clearly distinct from unfertilised pistils, and wild-type levels of seed-set per silique have previously been recorded for all single and double ga20ox mutants used in this analysis despite changes to silique length (Plackett et al. 2012). Where the presence of a single or a few seeds was suspected by distortion/bulging in apparently unpollinated pistils, and in cases of parthenocarpic pistil growth in response to GA treatment (VivianSmith and Koltunow 1999), these were checked manually by dissection. Silique-set was scored at each flower position between 1 and 10 (by which point, under our growth conditions, siliques were set reliably by all genotypes except ga20oxl ga20ox2 ga20ox3 and gal-3) for each genotype under control growth conditions and under exogenous GA treatment $(n=12)$.

\section{Characterisation of floral organ lengths and floral organ abnormalities across the early inflorescence}

Based on empirical evidence obtained from silique-set experiments, a range between flower positions 1-10 was selected for more detailed analysis of floral organ growth. Position 15 was also included as a representative of later flowering for comparison. These flower positions were sampled from wild-type and ga20ox mutant inflorescences under either control growth conditions or exogenous GA treatment $(n=4)$. Flowers were harvested from the primary inflorescence on the day of opening (floral stage 13; Smyth et al. 1990), taking a single flower per plant over the course of the experiment. In mutants where flower opening is not evident in the earliest flowers to develop (ga20ox1 ga20ox2 and ga20oxl ga20ox2 ga20ox3), the most advanced stage of development reached at each flower position was selected as a comparison. This was based on bud size, i.e. when the bud at the flower position specified had ceased to grow, and the following buds had attained a similar size, its development was adjudged to be complete. Upon harvesting, flowers were dissected and the number of floral organs in each whorl surveyed, noting the presence of any developmental abnormalities. Frequencies of deviations in floral organ number or organ developmental abnormalities were scored by type, with a value of 1 assigned for each individual instance observed. Dissected flowers were photographed, and the lengths of pistils, medial (long) stamens, petals and sepals were calculated from scaled images. Single measurements of each organ type were taken from each flower, measuring stamens, petals and sepals from where the organ joined the flower receptacle at its base to the organ tip. Pistils were measured from their base adjoining the receptacle to the lower edge of the stigma: a stamen length of $100 \%$ or greater relative to the pistil indicates that successful pollination is possible.

\section{Experimental design and statistical analysis}

Characterisation experiments were performed in blocked split-plot experimental designs (Gomez and Gomez 1984). GA treatment was applied to main plots (whole soil trays) and genotype to split plots (cells within soil trays). Siliqueset data were analysed using a generalised linear model fitting a logit link function (McCullagh and Nelder 1989) and assuming a binomial distribution. Pairwise comparisons of means in significant model terms $(p<0.05$, Chi-squared test) were made using individual least significant difference (LSD) values at a significance threshold of $1 \%$ (Online Resource 1). Floral organ growth was analysed both through ANOVA, utilising data from all flower positions, and linear regression modelling, for data from positions $1-10$. Where the distribution of data for individual floral organ types did not meet the assumptions of homogeneity of variance, analysis was performed on a transformed scale as required (ANOVA: square root for pistil and stamen data, Online Resource 2; linear regression: natural log for pistil and stamen data). For ANOVA, the standard error of the difference (SED) values on the relevant degrees of freedom was used to compare between genotypes at the same flower position, 
or between the different flower positions of one genotype within and between GA treatments, by way of the corresponding LSDs using a significance threshold of 5\%. For linear regression modelling, estimated parameters (slopes and intercepts of fitted lines) are presented with calculated 95\% confidence intervals (CIs). Comparisons of pairs of appropriate parameters between genotypes were made using $F$ tests.

Observed frequencies of floral abnormalities were found to approximate to a Poisson distribution and were analysed as such, fitting a generalised linear model (GLM) with a square-root link function (McCullagh and Nelder 1989). In the absence of over-dispersion, the main effects and interactions between the factors genotype, GA treatment and flower position were assessed using changes in model deviance ( $\chi^{2}$ tests). The predicted frequencies of abnormalities from this model were compared between genotypes within GA treatments, between GA treatments within genotypes and between flower positions within GA treatments using individual LSDs with a significance threshold of 5\% (Online Resource 3). The GenStat software package (2010, 13th edition; VSN International Ltd, Hemel Hempstead, UK) was used for all analyses.

\section{Results}

\section{A trend of increasing silique-set with advancing flower position was identified, irrespective of genotype or exogenous GA treatment}

As a proxy measure for floral organ development across early flower positions, the frequency of successful siliqueset (indicating successful pollination, which necessarily requires stamen growth to have matched the pistil) during early flowering of the primary inflorescence was compared between genotypes under both control growth conditions and exogenous GA treatment. It was found that, under our growth conditions, siliques were reliably being set by flower position 10 (the first flower to develop on the inflorescence representing position 1) in all genotypes except the severely GA-deficient mutants ga20oxl ga20ox2 ga20ox3 and gal-3 (Online Resource 4). It should be noted that a previous analysis of ga20oxl ga20ox2 fertility, Rieu et al. (2008) found silique-set defects up to approximately flower position 15 . We observed that there was no clear transition between early unpollinated flower positions and later successful siliqueset, i.e. a flower position with a developed silique could be followed by an unpollinated position (Online resource 4). In response to the stochastic nature of this phenotype, we analysed the mean frequencies of silique-set per flower position from 12 individual primary inflorescences for each genotype between flower positions 1-10.

GA20ox1, GA20ox2 and GA20ox3 were found to act together in a partially redundant manner to maintain siliqueset. Under control growth conditions, both the ga20oxl ga20ox2 ga20ox3 triple mutant and GA-deficient control (gal-3) did not set siliques across positions 1-10 (Fig. 1a), as previously found (Plackett et al. 2012). Whilst there were further significant quantitative differences between wild type (Col-0) and some ga20ox mutants in the frequency of silique-set averaged across flower positions 1-10 (ga20oxl and ga20ox1 ga20ox2, with silique-set reduced in both genotypes; Fig. 1a, $p<0.01$ ), there was no statistically significant interaction between genotype and flower position $(p=0.222)$, indicating that the pattern of silique-set across the early inflorescence was similar for all genotypes. In agreement with previous analyses of ga20ox mutant phenotypes (Rieu et al. 2008), under our experimental conditions mean silique-set was reduced more severely in ga20oxl ga20ox 2 than in ga20oxl $(p<0.01)$. The ga20oxl ga20ox3 and ga20ox2 ga20ox3 double mutants were found to phenocopy ga20oxl and ga20ox 2 , respectively ( $p>0.01$; Fig. 1a; with ga20oxl ga20ox3 also showing a significant reduction in fertility compared to wild type, $p<0.01$ ), suggesting that GA20ox3 acts redundantly with GA20oxl and GA20ox2 to promote successful silique-set. This same hierarchical relationship is also found across other plant developmental processes (Plackett et al. 2012).

GA treatment enhanced silique-set in the most severely GA-deficient genotypes (ga20oxl ga20ox2, ga20ox1 ga20ox2 ga20ox3 and gal-3) ( $p<0.01$; Fig. 1a), but reduced it in all other genotypes (including wild type) except ga20oxl $(p<0.01)$. Under GA treatment, mean silique-set became similar between all genotypes except gal-3 ( $p>0.01$; Fig. 1a). The observed negative effects of GA treatment on wild-type silique-set are consistent with previous studies (Rieu et al. 2008; Plackett et al. 2014), and the similarity in silique-set between most GA-treated genotypes indicates that GA responses were saturated under our exogenous treatment.

Irrespective of genotype, advancing flower position was found to have a significant positive effect on the probability of successful silique-set both under control growth conditions and under exogenous GA treatment (Fig. 1b). In both cases, the mean frequency of silique-set at flower 10 was significantly different compared to position $1(p<0.01)$. GA treatment reduced silique-set at all flower positions studied to a significant degree $(p<0.01)$ except position eight. This trend suggests that an additional mechanism is acting independently of GA signalling to increase the probability of silique-set as flowering progresses. Given the dependence of 

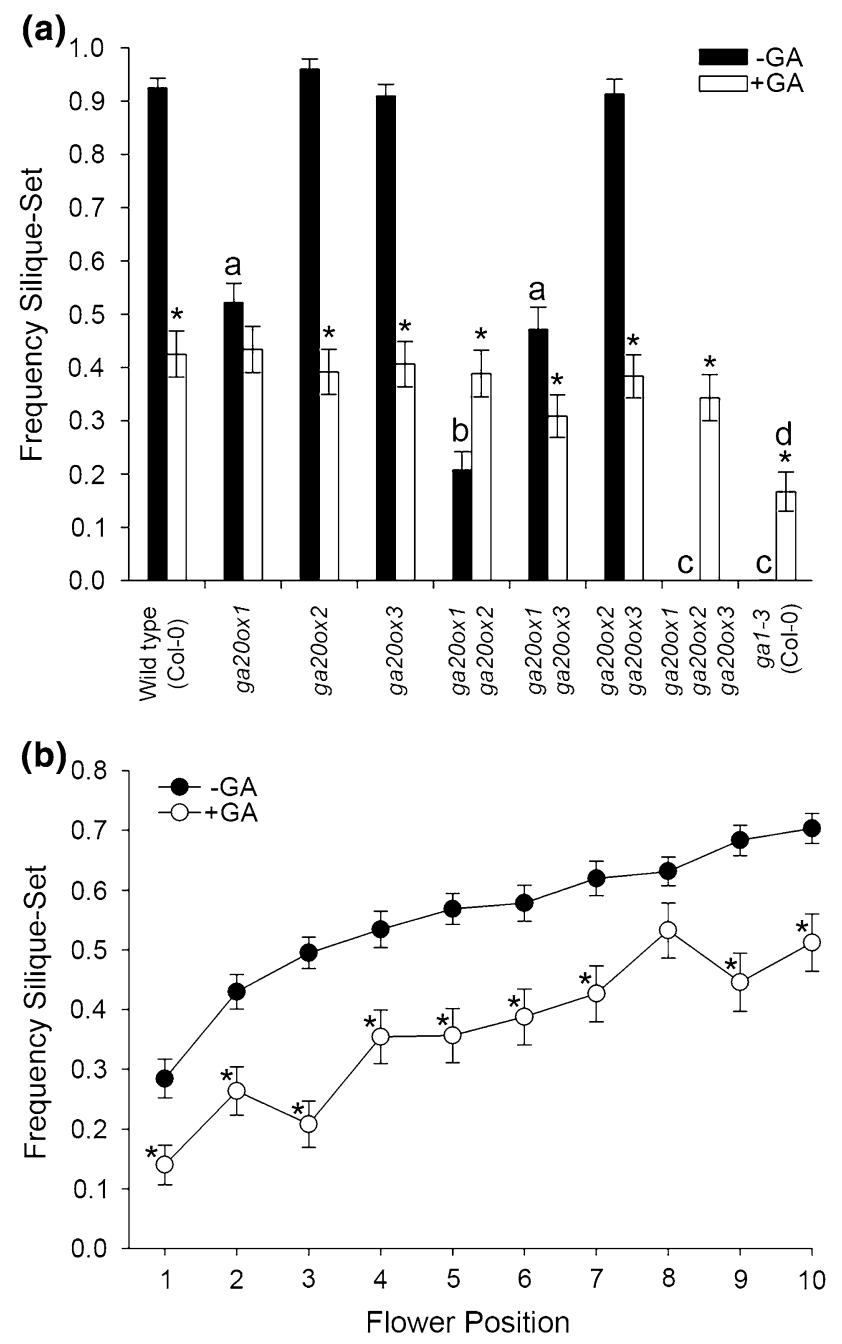

Fig. 1 Probability of Arabidopsis silique-set is position-dependent across flower positions 1-10. Statistically significant interactions were detected between genotype and GA treatment $(p<0.001)$ and between flower position and GA treatment $(p<0.001)$. a Mean silique-set frequencies in wild-type and ga20ox mutant inflorescences averaged across flower positions 1-10, under control growth conditions (black) and exogenous GA treatment (white). b Mean siliqueset frequencies for individual flower positions 1 through 10 averaged across all genotypes, under control growth conditions (black) and exogenous GA treatment (white). Values shown are the mean of 12 independent inflorescences \pm S.E. Pairwise comparisons between genotypes, GA treatments and flower positions were made using LSD values at a $1 \%$ significance threshold (See Online Resource 1). Letters denote significant difference $(p<0.01)$ from wild type under control growth conditions (black) or GA treatment (grey). Genotypes marked with different letters are significantly different from each other. Asterisks denote a significant effect of GA treatment within a genotype (a) or flower position (b)

pollination on coordinated floral organ growth, it was considered likely that this mechanism acts through differential changes to floral organ growth across early flowering.

\section{Changes in floral organ growth are sufficient to explain the recovery of silique-set in GA-deficient inflorescences}

To determine the contribution of floral organ growth to the changes in silique-set described above, we analysed ga20ox floral phenotypes (synchronised at flower opening, see "Materials and methods") between flower positions 1-10 under both control growth conditions and GA treatment. Comparing the phenotypes of the first (Fig. 2a-h) and tenth flowers (Fig. 2i-p) of each genotype showed that the greatest phenotypic changes across the early inflorescence occurred in ga20oxl ga20ox2: in flower 1 ga20oxl ga20ox2 stamens and petals were dramatically reduced compared to wild type (Fig. 2a, e) but were similar to wild type in flower 10 (Fig. 2i, j). The anthers in ga20oxl ga20ox2 flower 1 were indehiscent (Fig. 2e), whereas wild-type anthers had already released pollen by the time flower 1 opened. A similar phenotype of underdeveloped floral organs in early ga20oxl ga20ox 2 flowers was reported by Rieu et al. (2008). By the opening of flower 10 ga20oxl ga20ox 2 anthers had successfully dehisced, with pollen visible on the stigma (Fig. 2m). In contrast, ga20ox1 ga20ox2 ga20ox3 floral organs remained reduced and anthers indehiscent up to flower 10 (Fig. 2h, p). The floral phenotypes of all other genotypes were found to superficially resemble wild type. Interestingly, in most genotypes including wild type we observed that at flower opening stamens in flower 10 appeared longer relative to the pistil compared to flower 1 (Fig. 2a, i), suggesting that relative floral organ growth changes across early flowering. Spontaneous recovery of the ga20oxl ga20ox2 floral phenotype was thus confirmed over our selected experimental range. We also confirmed that under our experimental conditions exogenous GA treatment rescued the floral phenotypes of both ga20oxl ga20ox2 and ga20oxl ga20ox2 ga20ox3 to resemble that of wild type (Online Resource 4).

The hypothesis that changes in floral organ growth can explain the observed changes in silique-set was tested quantitatively through direct measurement of floral organs across the early inflorescence (Fig. 2q-v), comparing between genotypes at each flower position. For all genotypes, some stochastic variation in mean floral organ length was evident between adjacent flower positions (i.e. mean length could be lower or higher at a subsequent flower position), consistent with the observed variations in silique-set across the same range of flower positions.

In wild-type flowers, under control conditions mean stamen length was $\geq 100 \%$ of pistil length at flower opening at every flower position examined (Fig. 2q). Consistent with their silique-set phenotypes, a number of early flowers in both ga20oxl and ga20oxl ga20ox2 contained stamens whose relative length was significantly different from wild type and less than $100 \%$ of their respective pistils (Fig. 2q, 


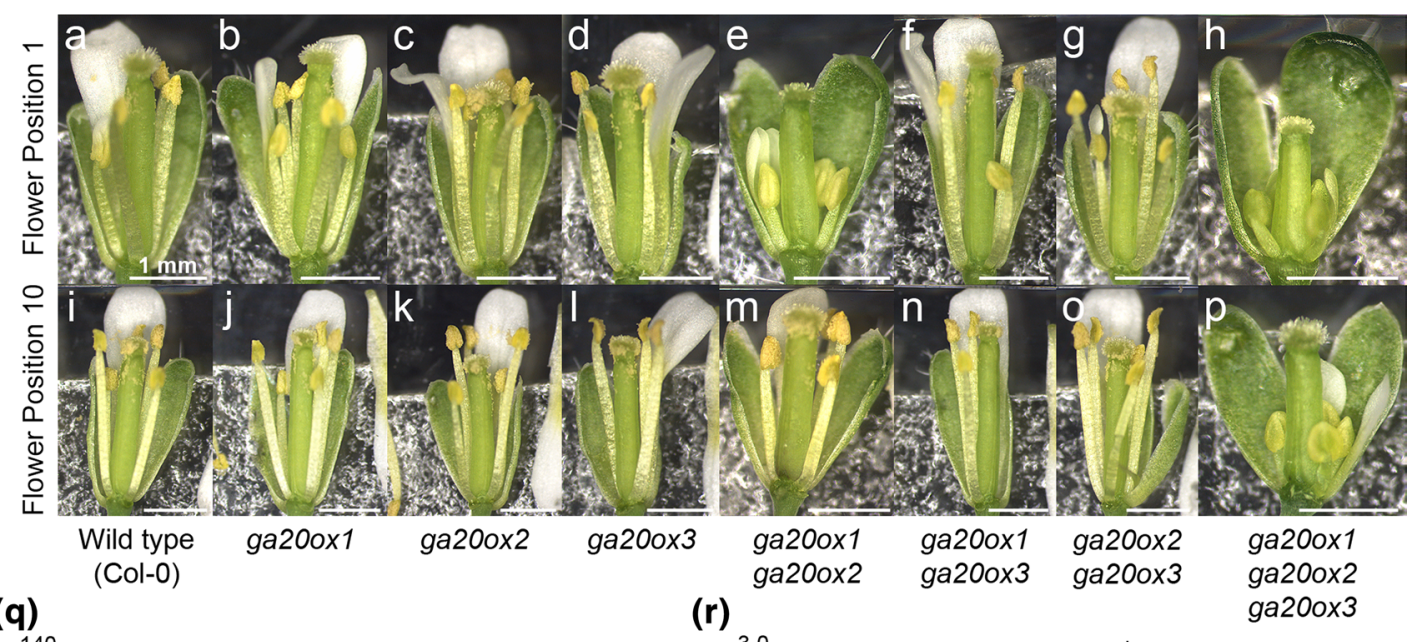

(q)

(r)

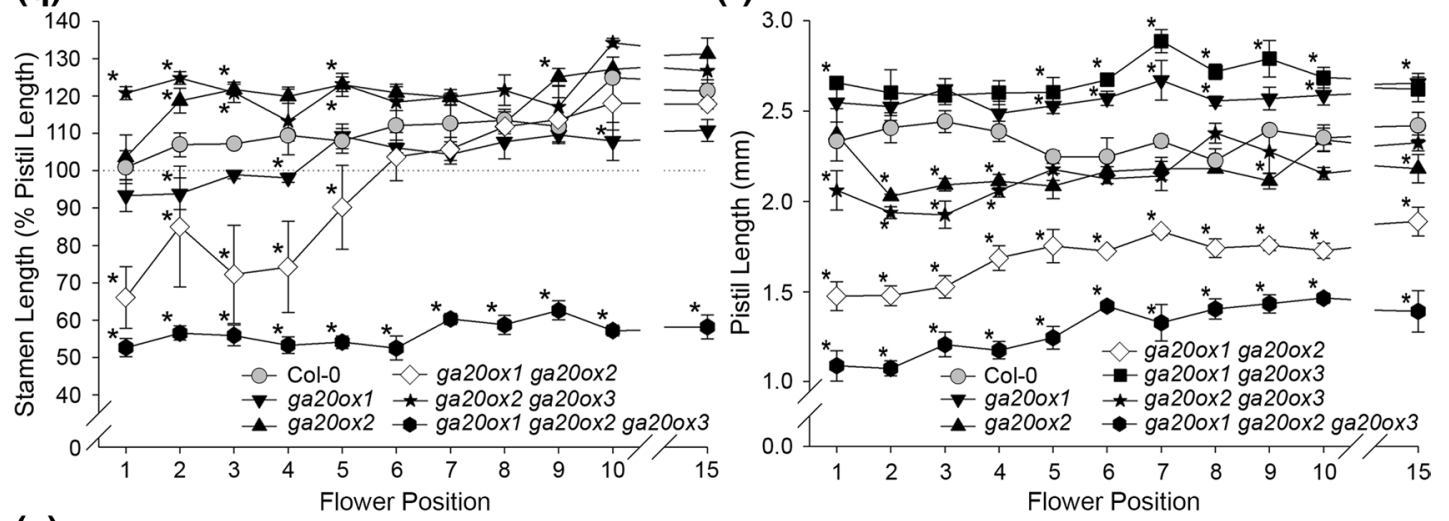

(s)

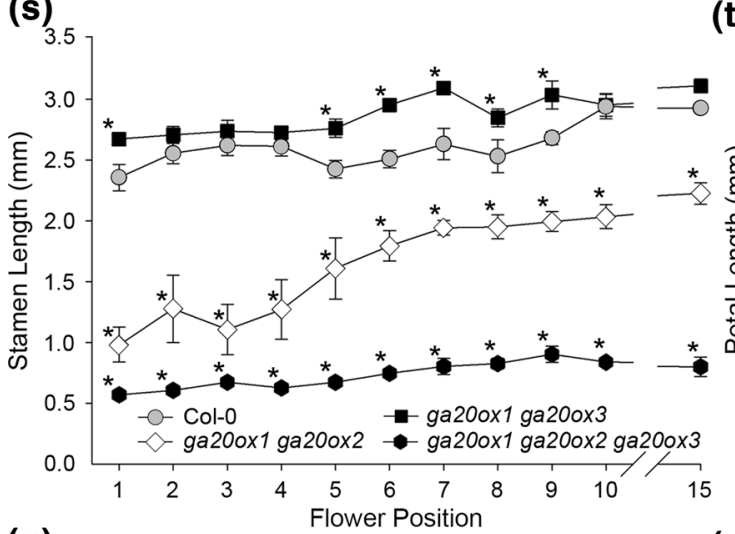

(t)

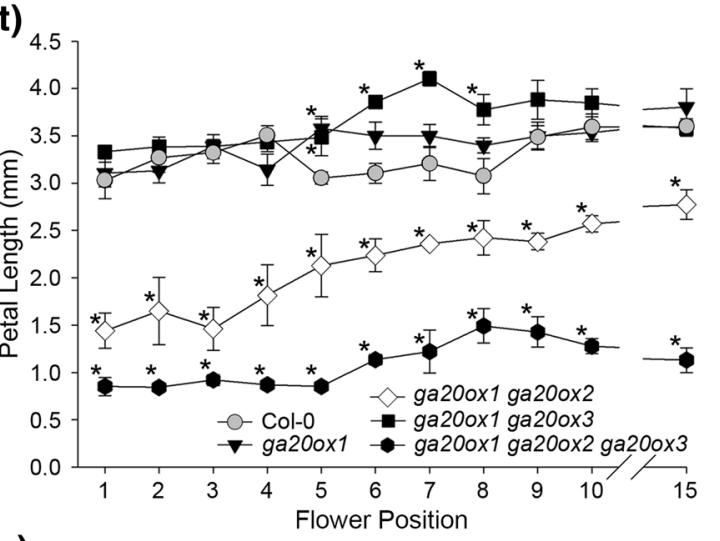

(u)

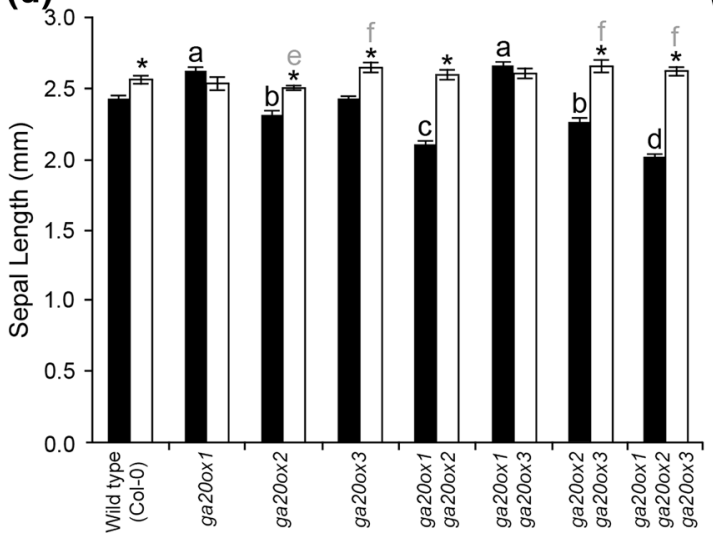

(v)

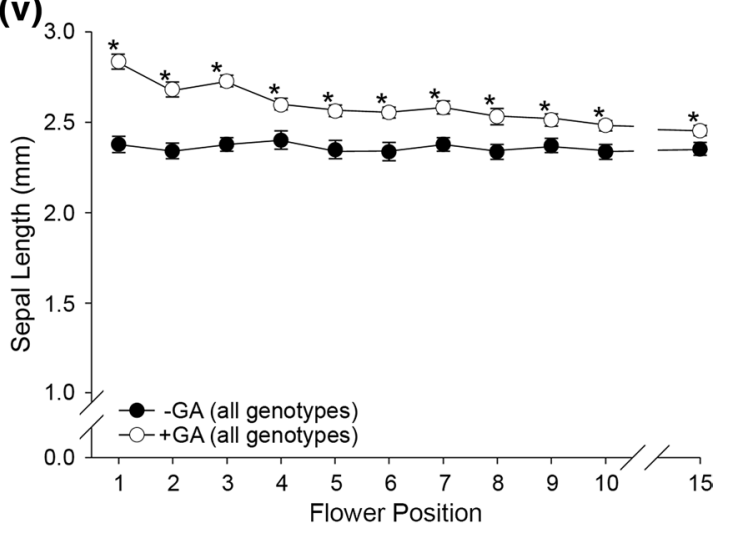


४Fig. 2 Arabidopsis floral organ lengths change in a position-dependent manner across flower positions 1-10. Floral phenotypes of wild type (Col-0) and ga20ox mutants, as specified, at flower positions one $(\mathbf{a}-\mathbf{h})$ and ten $(\mathbf{i}-\mathbf{p})$ under control growth conditions. All flowers shown are newly opened (floral stage 13; Smyth et al. 1990). Statistically significant three-way interactions were found between genotype, flower position and GA treatment for relative stamen length $(p<0.001)$ and absolute lengths of pistils $(p<0.001)$, stamens $(p<0.001)$ and petals $(p=0.002)$ across the early inflorescence. For sepals, a three-way interaction was not statistically significant $(p=0.275)$, but three separate significant two-way interactions were identified (genotype by GA, $p<0.001$; genotype by flower position, $p=0.018$; GA by flower position, $p<0.001$ ). Mean floral organ lengths of newly opened (stage 13) flowers across flower positions 1-10 and position 15, showing stamen length as a percentage of pistil length (q) and absolute lengths (in $\mathrm{mm}$ ) of pistils (r), stamens (s), petals (t). Flower position 15 is included as an indicator of floral organ growth in later flowering. Values shown are the mean of four independent flowers \pm S.E. Genotypes not represented on individual graphs were not significantly different from wild type at any flower position $(p>0.05)$. Significant differences $(p<0.05)$ from wild type within each flower position are denoted by asterisks. Mean sepal lengths of stage 13 flowers, for each genotype under control growth conditions (black) and exogenous GA treatment (white) averaged across all flower positions measured (u) and for each flower position under control growth conditions (black) and exogenous GA treatment (white), averaged across all genotypes (v). Values shown are the mean of $44(\mathbf{u})$ and 32 independent flowers $(\mathbf{v}) \pm$ S.E. Letters denote significant difference $(p<0.01)$ from wild type under control growth conditions (black) or GA treatment (grey). Genotypes marked with different letters are significantly different from each other. Asterisks denote a significant effect of GA treatment within a genotype (u) or flower position (v). Pairwise comparisons in (q-v) were made using LSDs at a significance threshold of 5\%, with pistil and stamen lengths being analysed on the square-root scale (see Online Resource 2)

$p<0.05)$. Congruent with their relative phenotypic severity, fewer flowers were significantly different between wild type and ga20oxl (flowers 2 and 4) than ga20oxl ga20ox2 (flowers 1-5), and at most of these earliest flower positions (flowers 1, 3, 4, 5) ga20oxl ga20ox2 relative stamen lengths were significantly reduced compared to ga20oxl $(p<0.05$; Online Resource 2). At later flower positions (beyond flower $5)$, stamen length in these two genotypes was not significantly different from wild type $(p>0.05)$ and consistently $\geq 100 \%$ of the pistil. In contrast to this, two other genotypes (ga20ox2 and ga20ox2 ga20ox3) surprisingly showed significant differences where relative stamen length was in fact greater than in wild type ( $p<0.05$; Fig. 2q). In ga20oxl ga20ox2 ga20ox3, relative stamen lengths were significantly different from both wild type and ga20oxl ga20ox2 at all flower positions examined ( $p<0.05$; Online Resource 2 ) and remained below $100 \%$ of pistil length throughout. Under GA treatment, relative stamen growth was rescued to $\geq 100 \%$ of the pistil in almost all genotypes and flower positions (Online Resource 4), indicating that the phenotypes observed are a result of impaired GA biosynthesis. These results suggest that the differences in mean silique-set observed between wild type and some GA-deficient mutants during early flowering (Fig. 1a) can mostly be explained through GA-dependent changes in stamen growth relative to the pistil in the earliest flowers to open, with all three GA20ox paralogues contributing.

Growth of all floral organs (as measured by absolute length at flower opening) was altered by GA deficiency, with loss of different paralogues having complex phenotypic effects. Consistent with the long-established role for GA in promoting Arabidopsis floral organ growth (Koornneef and Van der Veen 1980), ga20ox1 ga20ox2 ga20ox3 pistils, stamens and petals all exhibited significantly reduced growth compared to wild type at all flower positions $(p<0.05$; Fig. 2r-t), and mean sepal length across the early inflorescence was similarly reduced $(p<0.05$; Fig. $2 u$ ). Whilst the absolute length of all floral organs was also reduced in ga20oxl ga20ox 2 across the early inflorescence $(p<0.05$; Fig. $2 \mathrm{r}-\mathrm{u}$ ), floral organ growth was less severely reduced than the triple mutant phenotype ( $p<0.05$; Online Resource 2 ). In contrast to these two mutants, ga20oxl and ga20oxl ga20ox3 frequently demonstrated increased floral organ growth compared to wild type, with significantly different lengths in pistils ( $p<0.05$; Fig. 2r), stamens (ga20oxl ga20ox3 only) ( $p<0.05$; Fig. 2s), petals ( $p<0.05$; Fig. 2t) and sepals $(p<0.05$; Fig. $2 \mathrm{u})$. Loss of GA20ox2 alone, or in combination with GA20ox3, significantly affected pistil and sepal lengths ( $p<0.05$; Fig. 2 r, u), but not those of stamens or petals $(p>0.05)$. The relationship between GA20ox paralogues and growth of different floral organs across the early inflorescence was thus found to be complex, pointing to functional differences relating to their sites of expression. In particular, sepal responses appeared distinct from those of inner floral organs. These differences were also observed in flower 15 (Fig. 2r-t, v), suggesting that they are not limited to early flowering.

Importantly, ga20ox1 ga20ox2 floral organ lengths across the early inflorescence under control growth conditions show a clear trend of increasing stamen and petal length with advancing flower position (Fig. 2s, t). A similar trend might similarly be indicated in ga20ox 1 ga20ox2 pistils (Fig. 2r) but with a lesser magnitude than seen in stamens. This observation suggests the observed recovery of relative stamen growth in ga20oxl ga20ox2 might be explained by differentially increased growth of the stamen, whilst pistil growth remains relatively static. Our observations at the whole flower level (Fig. 2a-p) and significant increases in mean floral organ lengths between flowers 1 and 10 in other genotypes, including in wild type $(p<0.05$ for stamens and petals; Online Resource 2), suggest that changes in floral organ growth are a normal component of wild-type flowering.

GA treatment of ga20ox mutants rescued mean pistil, stamen and petal lengths to values similar to wild type at almost all organ, genotype and flower position 
Fig. 3 Effect of exogenous GA treatment on floral organ lengths of wild-type (Col-0) flowers during early flowering. Mean floral organ lengths of newly opened (stage 13) wild-type (Col-0) flowers across flower positions $1-10$ and position 15 under control growth conditions (-GA, black) and exogenous GA treatment (+GA, white), showing stamen length as a percentage of pistil length (a) and absolute lengths (in $\mathrm{mm}$ ) of pistils (b), stamens (c), petals (d) and sepals (e). Flower position 15 is included as an indicator of floral organ growth in later flowering. Values shown are the mean of four independent flowers \pm S.E. Significant differences $(p<0.05)$ between control growth conditions and GA treatment within each flower position are denoted by asterisks. Statistical analyses of stamens and petals were performed on a transformed scale (see "Materials and methods" and Online Resource 4). Statistical comparison of sepal lengths was not valid in the absence of a significant interaction between genotype, GA treatment and flower position (see Fig. 2)

combinations (Online Resource 4). Growth of wild-type pistils, stamens and petals was mostly unaffected by GA treatment across the early inflorescence (Fig. 3a-d). This is in contrast to our findings regarding silique-set, where under GA treatment successful wild-type silique-set was reduced to approximately $50 \%$ of that under control growth conditions (Fig. 1a). However, mean sepal length was significantly increased by GA treatment in all genotypes (including wild type) across the early inflorescence with the exception of ga20oxl and ga20ox1 ga20ox3 ( $p>0.05$; Fig. $2 \mathrm{u}$ ), in which sepal length was already significantly increased relative to wild type under control growth conditions ( $p<0.05$; Fig. $2 u$ ). Sepal length was also significantly increased under GA treatment at every flower position when averaged across all genotypes $(p<0.05$; Fig. 2v). We thus identified a differential response to GA between sepals and other floral organs. The observation that silique-set is reduced under exogenous GA treatment although stamen length being $\geq 100 \%$ of the pistil is consistent with previous observations of reduced fertility under GA treatment (Jacobsen and Olszewski 1993) and in constitutive GA signalling DELLA loss-of-function mutants (Dill and Sun 2001; Plackett et al. 2014), where GA-dependent defects in pollen development were identified as the cause.

\section{Floral organs display gradients of growth across the early inflorescence}

Linear regression modelling across flower positions 1-10 (see "Materials and methods") was used to test for the existence of significant changes in floral organ growth between different flowers of the early inflorescence. Growth of each floral organ type across the early inflorescence was thus described using two parameters: the intercept (a theoretical starting organ length at flower position 0) and the gradient (the change in floral organ length between two successive flower positions) (Table 1). (a)

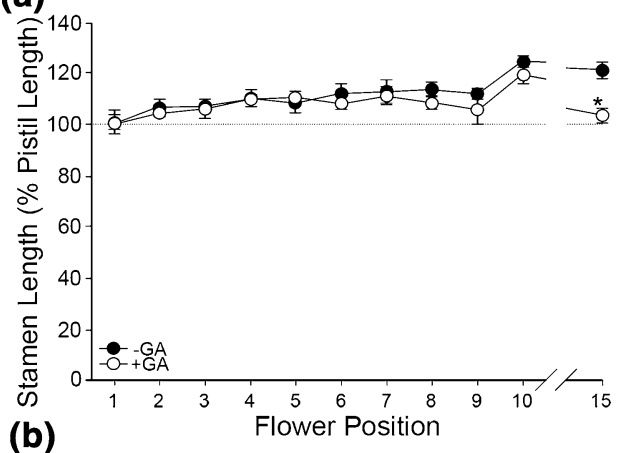

(b)

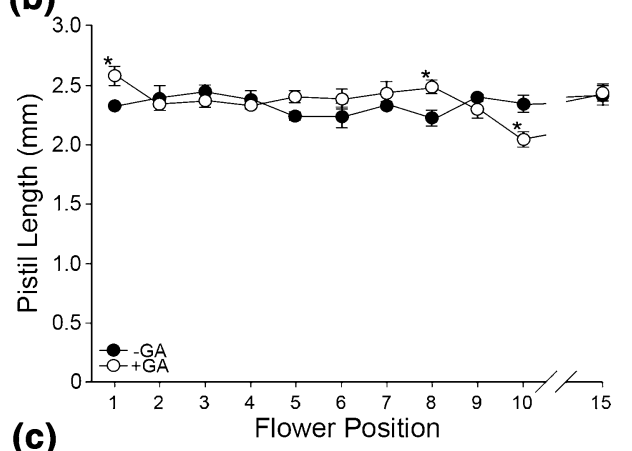

(c)

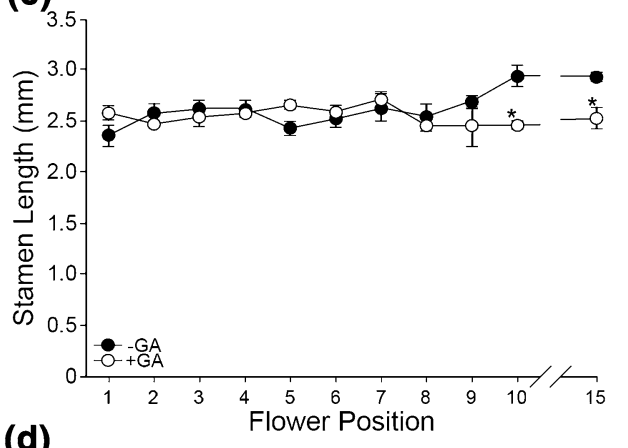

(d)

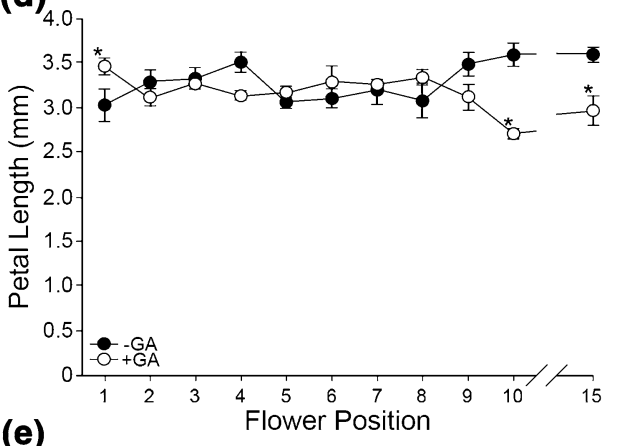

(e)

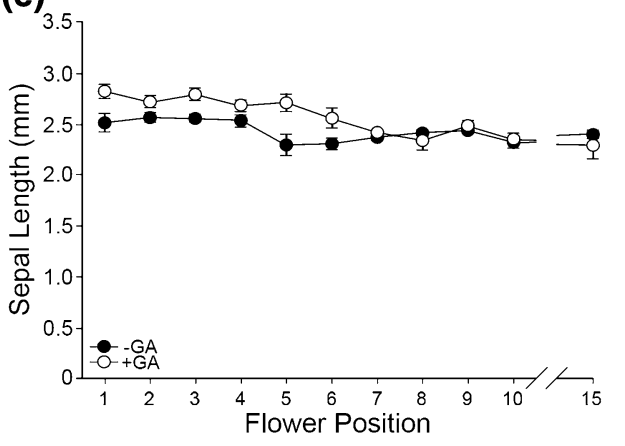


Under control growth conditions, we identified a significant positive gradient in stamen growth across the early wild-type inflorescence ( $p<0.05$; Table 1a), whereas the corresponding gradients for wild-type pistils and petals were not significantly different from $0(p>0.05)$. In contrast, a significant negative gradient growth was detected for sepals $(p<0.05)$. These results demonstrate the existence of previously unrecognised gradients in the growth of floral organs across the early inflorescence, which vary between different organ types. Differences in the gradient in growth between stamens and pistils can thus explain the positive trend in silique-set observed across the early inflorescence (Fig. 1b). No significant difference in the length of wild-type stamens was detected between flower positions 10 and 15 ( $p>0.05$, Online Resource 2), suggesting that this gradient could be limited to (or is at least strongest during) early flowering.

Under control growth conditions, a number of ga20ox mutants demonstrated altered floral organ growth parameters across the early inflorescence compared to wild type. All ga20ox mutants except ga20ox3 retained a significant positive gradient for stamen growth (significantly different from $0 ; p<0.05$, Table 1a). In the ga20oxl mutant, where relative stamen growth is reduced in the earliest flowers (Fig. 2q), stamen growth parameters were similar to wild type $(p>0.05)$, but initial pistil length (as described by the intercept) was significantly greater than in wild type $(p<0.05)$. In contrast, the ga20ox pistil gradient was not significantly different from wild type $(p>0.05)$. In consequence, reduced relative stamen growth in this genotype

Table 1 Linear regression modelling of floral organ lengths across the early Arabidopsis inflorescence

\begin{tabular}{|c|c|c|c|c|c|c|c|c|}
\hline Genotype & $\begin{array}{l}\text { Ln(Pistil) } \\
\text { intercept } \\
(-\mathrm{GA})\end{array}$ & $\begin{array}{l}\text { Ln(Pistil) } \\
\text { gradient } \\
(-\mathrm{GA})\end{array}$ & $\begin{array}{l}\mathrm{Ln} \text { (Stamen) } \\
\text { intercept } \\
(-\mathrm{GA})\end{array}$ & $\begin{array}{l}\text { Ln(Stamen) } \\
\text { gradient } \\
(-\mathrm{GA})\end{array}$ & $\begin{array}{l}\text { Petal } \\
\text { intercept } \\
(-\mathrm{GA})\end{array}$ & $\begin{array}{l}\text { Petal gradient } \\
(-\mathrm{GA})\end{array}$ & $\begin{array}{l}\text { Sepal } \\
\text { intercept } \\
(-\mathrm{GA})\end{array}$ & $\begin{array}{l}\text { Sepal gradient } \\
(-\mathrm{GA})\end{array}$ \\
\hline Wild type (Col-0) & [0.8639] & {$[-0.0030]$} & {$[0.8730]$} & {$[0.0133]$} & 3.1144 & 0.0275 & 2.5604 & -0.02445 \\
\hline ga20ox1 & {$[0.9282]^{\mathrm{a}}$} & [0.0024] & {$[0.8546]$} & [0.0204] & 3.1296 & 0.0452 & $2.7309^{\mathrm{a}}$ & -0.01884 \\
\hline ga20ox2 & {$[0.7523]^{\mathrm{b}}$} & [0.0034] & {$[0.8590]$} & {$[0.0172]$} & 3.0995 & 0.0300 & $2.2452^{b}$ & $0.01101^{\mathrm{a}}$ \\
\hline ga20ox3 & [0.8589] & {$[-0.0064]$} & [0.9044] & [0.0097] & 3.1680 & 0.0213 & 2.4997 & -0.01264 \\
\hline ga20ox1 ga20ox2 & {$[0.3903]^{\mathrm{c}}$} & {$[0.0216]^{\mathrm{a}}$} & {$[-0.0945]^{\mathrm{a}}$} & {$[0.0914]^{\mathrm{a}}$} & $1.3181^{\mathrm{a}}$ & $0.1325^{\mathrm{a}}$ & $2.0323^{\mathrm{c}}$ & $0.01216^{\mathrm{a}}$ \\
\hline ga20oxl ga20ox3 & {$[0.9450]$} & {$[0.0071]^{\mathrm{b}}$} & {$[0.9670]^{\mathrm{b}}$} & {$[0.0141]$} & 3.2349 & $0.0753^{\mathrm{b}}$ & $2.6641^{\mathrm{a}}$ & $-0.00051^{\mathrm{a}}$ \\
\hline ga20ox2 ga20ox3 & {$[0.6606]^{\mathrm{b}}$} & {$[0.0162]^{\mathrm{a}}$} & {$[0.8296]$} & {$[0.0202]$} & 2.9798 & 0.0527 & $2.1825^{\mathrm{b}}$ & $0.01287^{\mathrm{a}}$ \\
\hline $\begin{array}{l}\text { ga20oxl ga20ox } 2 \\
\text { ga20ox3 }\end{array}$ & {$[0.0436]^{\mathrm{d}}$} & {$[0.0362]^{\mathrm{c}}$} & {$[-0.6024]^{c}$} & {$[0.0487]^{\mathrm{b}}$} & $0.6830^{\mathrm{b}}$ & $0.0737^{b}$ & $2.0175^{\mathrm{d}}$ & -0.00105 \\
\hline \pm for $95 \%$ C.I. & {$[0.0481]$} & {$[0.0078]$} & {$[0.0672]$} & {$[0.0108]$} & 0.1942 & 0.0312 & 0.0870 & 0.01298 \\
\hline \multicolumn{9}{|l|}{ (b) } \\
\hline Genotype & $\begin{array}{l}\text { Ln(Pistil) } \\
\text { intercept } \\
\text { (+GA) }\end{array}$ & $\begin{array}{l}\text { Ln(Pistil) } \\
\text { gradient } \\
(+\mathrm{GA})\end{array}$ & $\begin{array}{l}\text { Ln(Stamen) } \\
\text { intercept } \\
(+\mathrm{GA})\end{array}$ & $\begin{array}{l}\text { Ln(Stamen) } \\
\text { gradient } \\
(+\mathrm{GA})\end{array}$ & $\begin{array}{l}\text { Petal } \\
\text { intercept } \\
(+\mathrm{GA})\end{array}$ & $\begin{array}{l}\text { Petal gradient } \\
(+\mathrm{GA})\end{array}$ & $\begin{array}{l}\text { Sepal } \\
\text { intercept } \\
(+\mathrm{GA})\end{array}$ & $\begin{array}{l}\text { Sepal gradient } \\
(+\mathrm{GA})\end{array}$ \\
\hline Wild type (Col-0) & {$[0.9233]$} & {$[-0.0111]$} & {$[0.9440]$} & {$[-0.0005]$} & 3.3775 & $-0.0362 *$ & $2.8789 *$ & $-0.05398 *$ \\
\hline ga20oxl & {$[0.9070]$} & {$[-0.0099]^{*}$} & {$[0.8853]$} & {$[0.0044]^{*}$} & 3.1862 & $-0.0358^{*}$ & $2.7967 *$ & $-0.04837 *$ \\
\hline ga20ox2 & {$[0.8356]^{\mathrm{a} *}$} & {$[-0.0034]$} & {$[0.9107]^{*}$} & [0.0029] & 3.1416 & -0.0049 & $2.6190^{\mathrm{a} *}$ & $-0.01852^{\mathrm{a} *}$ \\
\hline ga20ox3 & {$[0.8380]$} & {$[0.0045]^{\mathrm{a}}$} & {$[0.9210]$} & {$[0.0052]$} & 3.2390 & -0.0017 & $2.8923^{*}$ & $-0.04217 *$ \\
\hline ga20ox1 ga20ox2 & {$[0.8460]^{*}$} & {$[-0.0015]^{*}$} & {$[0.8396]^{\mathrm{a} *}$} & {$[0.0091]^{*}$} & $3.1806^{*}$ & $0.0064^{\mathrm{a} *}$ & $2.6933^{\mathrm{a} *}$ & $-0.01737^{\mathrm{a} *}$ \\
\hline ga20ox1 ga20ox3 & [0.9196] & {$[-0.0038]$} & [0.9019] & {$[0.0100]$} & 3.2915 & $-0.0040^{*}$ & $2.7725^{*}$ & $-0.03004^{\mathrm{b} *}$ \\
\hline ga20ox2 ga20ox3 & {$[0.8875]^{*}$} & {$[-0.0008]^{*}$} & {$[0.8852]$} & {$[0.0095]$} & 3.2821 & $-0.0051^{*}$ & $2.7590 *$ & $-0.01666^{\mathrm{ab} *}$ \\
\hline $\begin{array}{l}\text { ga20ox1 ga20ox } 2 \\
\text { ga20ox3 }\end{array}$ & {$[0.9033]^{*}$} & {$[-0.0093]^{*}$} & {$[0.8711]^{a *}$} & {$[0.0021]^{*}$} & $3.1326^{\mathrm{a} *}$ & $-0.0118^{*}$ & $2.7891 *$ & $-0.03058^{\mathrm{b} *}$ \\
\hline \pm for $95 \%$ C.I. & {$[0.0481]$} & {$[0.0078]$} & {$[0.0672]$} & {$[0.0108]$} & 0.1942 & 0.0312 & 0.0870 & 0.01300 \\
\hline
\end{tabular}

Linear regression modelling of floral organ lengths in stage 13 Arabidopsis flowers across flower positions 1-10 under control growth conditions (a) and exogenous GA treatment (b) (see "Materials and methods"). The linear models fitted accounted for a significant proportion of variance ( $p<0.001$ ): $R^{2}=86 \%$ (pistils), 92\% (stamens), $81.7 \%$ (petals) and 63.6\% (sepals) of variance, respectively. Values shown are estimated intercepts (organ length in $\mathrm{mm}$ at theoretical flower position 0 ) and gradients (change in organ length in mm with flower position) given four independent flowers per genotype per flower position, and the \pm adjustment for $95 \%$ confidence intervals (CI). Where analysis was performed on transformed data to fit statistical assumptions (see "Materials and methods"), these values are given in square brackets. Superscript letters denote significant difference from the wild type $(p<0.05)$. Genotypes marked with different superscript letters are significantly different from one another. Asterisks indicate a significant effect of GA treatment $(p<0.05)$ compared to control growth conditions 
does not relate to changes in stamen growth but is instead the outcome of increased pistil growth throughout the early inflorescence, delaying the flower position in which stamen growth becomes sufficient to ensure pollination. Conversely in ga20ox2, which demonstrated increased relative stamen growth in some early flowers (Fig. 2q), the pistil intercept was reduced compared to wild type $(p<0.05)$, whilst the pistil gradient and stamen parameters were not significantly different from wild type $(p>0.05)$. Loss of GA20ox3 in either the ga20oxl or ga20ox2 backgrounds caused further significant changes in growth relationships $(p<0.05$; Table 1a), suggesting that these paralogues are not fully redundant. Modelling growth relationships at the level of the inflorescence rather than individual flowers has thus provided a better description of the processes underlying changes in relative organ growth, and through this a clearer understanding of GA20ox paralogue function.

In the ga20oxl ga20ox2 background, pistil, stamen and petal growth parameters were all significantly different from those of wild-type, ga20ox 1 and ga20ox $2(p<0.05$; Table 1a). Growth parameters for these organs also differed between ga20ox1 ga20ox2 $(p<0.05)$ and ga20ox1 ga20ox2 ga20ox3. In these two more severely GA-deficient genotypes, in addition to the stamen gradient, pistil and petal gradients also became significantly positive $(p<0.05)$. Sepal growth was also positive in ga20ox 1 ga20ox $2(p<0.05)$, but not in the triple mutant $(p>0.05)$. In conjunction with these positive gradients, floral organ intercept values were all reduced in these two mutants $(p<0.05)$, reflecting shorter organs in the first flowers to open followed by an increased rate of organ growth with advancing flower position compared to wild type. These results suggest that the positive gradient in stamen growth seen under control growth conditions could be independent of GA biosynthesis and that GA-dependent floral organ growth is overlaid onto other, independent gradients.

Under GA treatment, the growth relationships of floral organs in ga20ox mutants were mostly rescued to that of wild type (Table 1b). In this analysis, the wild-type inflorescence did show some response to GA treatment, with significant changes in the growth gradients of petals and sepals $(p<0.05)$, and a significant increase in the sepal intercept $(p<0.05)$. Whilst there was no formal significant difference in wild-type stamens and pistils between control growth conditions and under GA treatment, the stamen gradient under GA treatment was found to be no longer significantly different from 0 . In all GA-treated genotypes, the gradients of each floral organ type (including stamens) were either not significantly different from $0(p>0.05)$ or negative $(p<0.05)$ (Table $1 \mathrm{~b})$. This suggests that floral organ growth responses to GA are not fully saturated in early flowers. Changes in GA biosynthesis or signalling may therefore play a role in the early wild-type inflorescence to generate organ-specific growth gradients.

\section{The recovery of ga200x1 ga20ox2 stamen development can be explained through a discontinuous phenotype independent of gradients in organ growth}

Stamen length relative to the pistil in the ga20ox1 ga20ox 2 mutant was found to be far more variable than in other genotypes in the earliest flowers (flower positions 1-5; Fig. 2q). This was caused by variation in the absolute lengths of stamens, but not of pistils (Fig. 2r, s). It was noted that stamens of ga20ox1 ga20ox2 flowers were also frequently indehiscent in this region of the early inflorescence, but not beyond. To test for a relationship between these two factors, the lengths of floral organs from ga20ox1 ga20ox2 flowers with indehiscent or dehiscent anthers were plotted separately (Fig. 4). Whilst ga20ox1 ga20ox2 pistils were of similar lengths between flowers with dehiscent and indehiscent anthers (Fig. 4a), stamens with indehiscent anthers were clearly further reduced in length (Fig. 4b). ga20ox1 ga20ox2 flowers with indehiscent anthers also showed a reduction in petal length (Fig. 4c), but not sepal length (Fig. 4d). Failure in anther dehiscence in early ga20ox1 ga20ox2 flowers thus appears to be specifically associated with reduced growth of particular floral organs (stamen and petals) and not others (pistils and sepals).

To determine whether reduced stamen and petal growth in flowers with indehiscent anthers were sufficient to explain the significant differences in growth parameters between ga20ox1 ga20ox2 and wild-type inflorescences (Table 1a), linear regression analysis was repeated for stamens and petals excluding measurements from indehiscent flowers (Table 2). With indehiscent organ lengths excluded, the difference in the gradient between ga20ox 1 ga20ox 2 and wildtype stamens became much less significant $(p<0.001$ to $p=0.036$ ), and the gradient of petal growth became similar $(p=0.262)$. Both gradient values remained significantly different from 0 ( $p<0.05$, Table 2$)$. The ga20ox1 ga20ox 2 stamen and petal intercept values remained significantly different from wild type ( $p<0.001$ for each), reflecting reduced organ growth at all flower positions. Much of the dramatic 'recovery' phenotype seen across the early ga20ox1 ga20ox2 inflorescence can therefore be explained by a separable phenotype in which stamen and petal growth are restricted in flowers exhibiting indehiscent anthers. Underlying this, an independent gradient of increasing stamen and petal length with advancing flower position remains. 
Fig. 4 ga20ox1 ga20ox2 stamen and petal lengths are reduced in flowers with indehiscent anthers. Distribution of organ lengths for pistils (a), stamens (b), petals (c) and sepals (d) from ga20oxl ga20ox 2 flowers across the early inflorescence, distinguishing flowers where anthers were indehiscent (white) or dehiscent (black) at flower opening. Wild-type floral organ lengths (in which all flowers were dehiscent) are shown in grey for comparison

\section{Floral patterning is not stereotypical in the first flowers to develop}

Although Arabidopsis floral development is reported as highly stereotypical (Smyth et al. 1990), we frequently observed deviations from this pattern, with abnormal numbers of floral organs present and/or developmental defects in individual organs. These abnormalities were found in all genotypes including wild type (Fig. 5a). Two mutants, ga20oxl and ga20oxl ga20ox3, demonstrated mean frequencies of abnormalities significantly different from wild type ( $p<0.05$; Fig. 5a). Surprisingly, these mutants demonstrated apparently opposing phenotypes with a reduced and increased frequency of abnormalities, respectively. In contrast, the two most GA-deficient mutants, ga20oxl ga20ox2 and ga20oxl ga20ox2 ga20ox3, did not demonstrate significant differences from wild type $(p>0.05)$. When plotted by flower position, the mean frequency of abnormalities in wild type under control growth conditions was greatest across flowers $1-3$ before dropping away (Fig. 5b). Abnormalities in ga20oxl were reduced in this region, but whereas ga20oxl ga20ox3 displayed a similar frequency of abnormalities to wild type in the earliest flowers, they continued to appear in later flowers to develop. Under control growth conditions, floral abnormalities were most frequent in the first flowers to develop in all genotypes, with the mean frequency across all genotypes highest in flower 1 and then reducing significantly by flowers $3-4$ ( $p<0.05$; Fig. 5 c). The frequency of abnormalities in these first flowers was not affected by GA treatment, but were significantly more frequent than under control growth conditions by flower position $4(p<0.05)$ and they became increasingly frequent as flowering progressed.

It thus appears that, contrary to previous assumptions, Arabidopsis floral patterning is not uniformly stereotypical across the inflorescence, with the first flowers to develop showing significant deviations and patterning not becoming fully constrained until flower four. Differences in phenotype between ga20oxl and ga20oxl ga20ox3 suggest a possible role for GA, strongly supported by the GA treatment results. The maintenance of developmental abnormalities into later flowering under GA treatment suggests that a restriction of GA biosynthesis or signalling is involved in the imposition of stereotypical floral development beyond the first flowers.
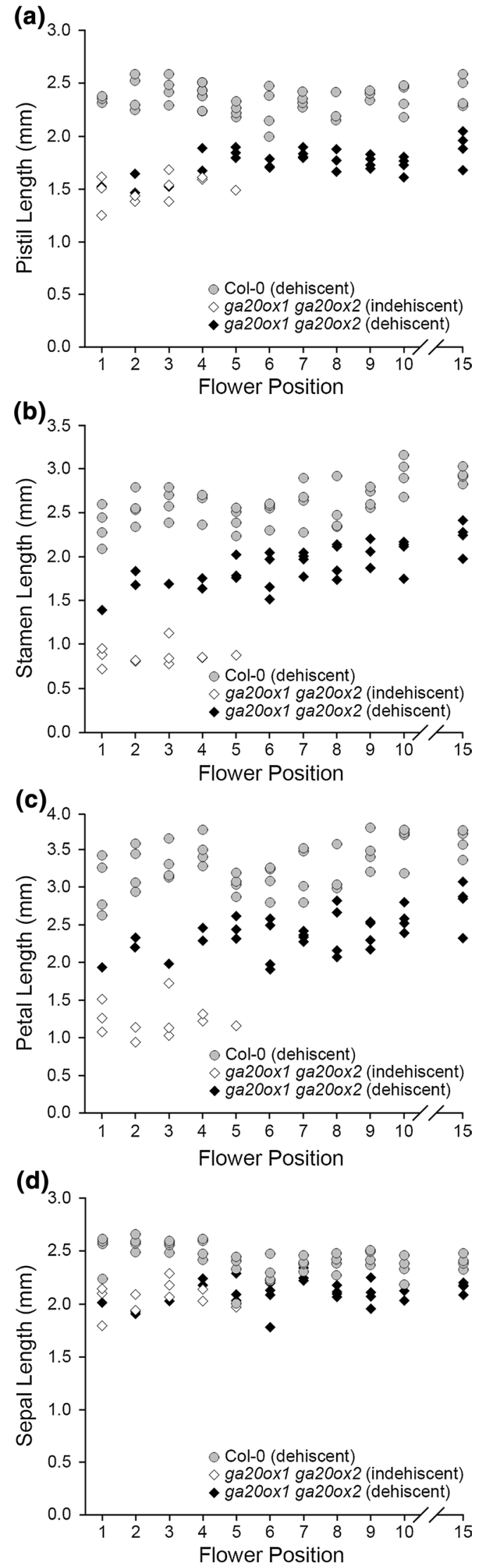
Table 2 Excluding indehiscent floral organs from ga20ox1 ga20ox2 results in growth relationships similar to wild type

\begin{tabular}{|c|c|c|c|c|c|c|c|c|}
\hline Genotype & $\begin{array}{l}\text { Ln(Stamen) } \\
\text { intercept (- } \\
\text { GA) }\end{array}$ & $\begin{array}{l}\text { Ln(Stamen) } \\
\text { gradient (- } \\
\text { GA) }\end{array}$ & $\begin{array}{l}\text { Ln(Stamen) } \\
\text { intercept } \\
(+\mathrm{GA})\end{array}$ & $\begin{array}{l}\text { Ln(Stamen) } \\
\text { gradient } \\
(+\mathrm{GA})\end{array}$ & $\begin{array}{l}\text { Ln(Petal) } \\
\text { intercept (- } \\
\text { GA) }\end{array}$ & $\begin{array}{l}\text { Ln(Petal) } \\
\text { gradient (- } \\
\text { GA) }\end{array}$ & $\begin{array}{l}\text { Ln(Petal) } \\
\text { intercept } \\
\text { (+GA) }\end{array}$ & $\begin{array}{l}\text { Ln(Petal }) \\
\text { gradient } \\
(+\mathrm{GA})\end{array}$ \\
\hline Wild type (Col-0) & {$[0.8730]$} & {$[0.0133]$} & {$[0.9440]$} & {$[-0.0005]$} & [1.1331] & [0.0083] & [1.2191] & {$[-0.0120]$} \\
\hline ga20oxl & {$[0.8546]$} & {$[0.0204]$} & {$[0.8853]$} & {$[0.0044]$} & [1.1379] & {$[0.0138]$} & {$[1.1565]$} & {$[-0.0120]$} \\
\hline ga20ox 2 & {$[0.8590]$} & {$[0.0172]$} & [0.9107] & [0.0029] & [1.1309] & [0.0089] & {$[1.1423]$} & {$[-0.0015]$} \\
\hline ga20ox3 & [0.9044] & [0.0097] & {$[0.9210]$} & {$[0.0052]$} & [1.1469] & [0.0067] & [1.1727] & {$[-0.0007]$} \\
\hline ga20ox1 ga20ox2 & {$[0.4345]^{\mathrm{a}}$} & {$[0.0285]^{\mathrm{a}}$} & {$[0.8396]^{\mathrm{a}}$} & {$[0.0091]$} & {$[0.7316]^{\mathrm{a}}$} & {$[0.0184]$} & [1.1509] & {$[0.0025]^{\mathrm{a}}$} \\
\hline ga20ox1 ga20ox3 & {$[0.9670]$} & {$[0.0141]$} & [0.9019] & {$[0.0100]$} & {$[1.1770]$} & [0.0206] & [1.1849] & {$[-0.0009]$} \\
\hline ga20ox2 ga20ox3 & [0.8296] & {$[0.0202]$} & {$[0.8852]$} & {$[0.0095]$} & [1.0914] & [0.0159] & [1.1864] & {$[-0.0018]$} \\
\hline $\begin{array}{l}\text { ga20ox1 ga20ox } 2 \\
\text { ga20ox3 }\end{array}$ & {$[-0.6024]^{\mathrm{b}}$} & {$[0.0487]^{\mathrm{b}}$} & {$[0.8711]^{\mathrm{a}}$} & {$[0.0021]$} & {$[-0.3146]^{\mathrm{b}}$} & {$[0.0658]^{\mathrm{a}}$} & {$[1.1364]^{\mathrm{a}}$} & {$[-0.0036]$} \\
\hline \pm for $95 \% \mathrm{CI}$ & {$[0.0528]$} & {$[0.0085]$} & {$[0.0528]$} & {$[0.0085]$} & {$[0.0660]$} & {$[0.0106]$} & {$[0.0660]$} & {$[0.0106]$} \\
\hline
\end{tabular}

Re-analysis of linear regression modelling of stamen and petal lengths in newly opened Arabidopsis flowers across flower positions 1-10 under control growth conditions (see "Materials and methods"), excluding values from ga20oxl ga20ox2 flowers where stamens were indehiscent at flower opening (Fig. 4b, c). Removal of data from indehiscent flowers marginally increased the explanatory power of both models: $R^{2}=93 \%$ (stamens) and $85.8 \%$ (petals) of variance, respectively. Values shown are estimated intercepts (organ length in mm at theoretical flower position 0 ) and gradients (change in organ length in mm with flower position) and the \pm adjustment for $95 \%$ confidence intervals (CI). Parameter estimates for ga20ox1 ga20ox2 are highlighted in bold. The analysis was performed on transformed data to fit statistical assumptions (see "Materials and methods") so the values are given in square brackets. Superscript letters denote significant difference from the wild type ( $p<0.05)$. Genotypes marked with different superscript letters are significantly different from one another

\section{GA-dependent effects on floral patterning were strongest in stamens}

Defects in flower morphology are likely to have arisen early in floral development, pointing to previously unrecognised functions for GA during this stage. To specify these possible functions more closely, the observed floral abnormalities were subdivided into phenotypic classes and by floral organ type: sepals (whorl 1, outermost), petals (whorl 2), stamens (whorl 3) and the pistil (whorl 4, innermost). Given that lateral (or 'short') stamens within whorl three arise separately, after the emergence of medial (or 'long') stamens (Smyth et al. 1990), long and short stamens were treated as separate organ types. The phenotypic classes identified comprised deviations in the numbers of each floral organ type (gain or loss of organs relative to the expected number; $83.61 \%$ of abnormalities over all genotypes); organ fusion or splitting, here defined as the emergence of two partially or wholly fused organs from the same position within the floral plan $(10.16 \%)$ and partial homeotic conversion of organ identity $(6.23 \%)$.

To better understand the factors underlying these different types of abnormality, the relative contributions of genotype, flower position and GA treatment were re-assessed using GLM (see "Materials and methods"). When analysing the frequency of deviations from the expected number of floral organs across all floral organ types (whole flowers), a significant interaction was found only between GA treatment and flower position ( $p<0.001$; Table 3 ), whilst genotype was a significant independent factor $(p<0.001)$. Within individual organ types, only individual factors remained independently significant (Table 3). Similarly, no significant two- or threeway interactions were detected in relation to frequencies of floral organ fusion or homeosis for either whole flowers or individual organs $(p>0.05)$, but single factors remained significant $(p<0.05$; Table 3$)$. The interactions detected between experimental treatments when considering all floral abnormalities together and at the scale of the whole flower are thus likely to represent a combination of multiple independent phenotypic effects occurring at a smaller scale. GA treatment significantly affected the numbers of all floral organ types $(p<0.05)$ and the frequency of organ fusion events. In contrast, organ homeosis was not affected by GA treatment $(p=0.378)$. The effects of genotype were specific to short stamens, both in their number $(p=0.006)$ and the occurrence of homeotic events $(p=0.007)$. Flower position had a significant effect only on the numbers of both long and short stamens ( $p=0.007$ and $p<0.001$, respectively). Thus, the effect of these factors on floral development can be interpreted more closely.

Changes observed in the number of floral organs were not uniform: while only additional organs were detected for sepals, both organ loss and gain were observed for petals and both stamen types (see Online Resource 5). Under control growth conditions, deviations in the number of floral organ occurred most frequently in long and short stamens (Fig. 6a). GA treatment significantly increased the frequency of deviations in the numbers of sepals, petals and short stamens ( $p<0.05$; Fig. 6a). Only increases in net sepal number were recorded under GA treatment, whereas short stamens 
Fig. 5 Early floral developmental events are significantly affected by both GA signalling and flower position. The incidence of floral abnormalities was analysed by GLM (see "Materials and methods"). Statistically significant two-way interactions were detected between genotype and GA treatment $(p=0.024)$, genotype and flower position $(p=0.033)$ and between GA treatment and flower position $(p<0.001)$. There was no significant three-way interaction $(p=0.807)$. a Mean frequencies of floral abnormalities (collective deviations in expected floral organ number, organ fusion and organ homeosis) in ga20ox mutants averaged across the early inflorescence (flowers 1-10), under control (black) and GA-treated conditions (white). Values shown are the mean of 44 independent flowers \pm S.E. Asterisks indicate a significant difference between control and GAtreated conditions $(p<0.05)$ within a genotype. Letters indicate significant difference $(p<0.05)$ of genotypes compared to wild type under either control growth conditions (black letters) or GA treatment (grey letters). Genotypes or flower positions denoted by different letters are significantly different from one another $(p<0.05)$. b Plotted mean frequencies of floral abnormalities at flower positions 1-10 and 15 under control growth conditions, comparing wild type to the ga20oxl and ga20oxl ga20ox3 mutants which showed significant differences in (a). Values shown are the mean of four independent flowers \pm S.E. c Mean frequencies of floral abnormalities at flower positions $1-10$ and 15 averaged across all genotypes, under control (black) and GA-treated conditions (white). Values shown are the mean of 32 independent flowers \pm S.E. Asterisks indicate a significant difference between control and GA-treated conditions $(p<0.05)$ within a single flower position. Letters indicate significant difference $(p<0.05)$ of flower positions compared to position one under either control growth conditions (black letters) or GA treatment (grey letters). Flower positions denoted by different letters are significantly different from one another $(p<0.05)$ within the same growth condition. No comparison was made between different flower positions from separate growth conditions. Pairwise comparisons in $(\mathbf{a}, \mathbf{c})$ were made using LSDs with a significance threshold of 5\% (see Online Resource 3)

were almost always lost. Losses and gains of long stamens were detected under both growth conditions (see Online Resource 5). Deviations in the numbers of both long and short stamens occurred most frequently in the first flowers to open (Fig. 6b). Under GA treatment, more deviations in the numbers of short stamens occurred across the early inflorescence (Fig. 6b), although these appeared increasingly frequent in later flowers. The effect on the numbers of long stamens was much less pronounced. These changing distributions of floral organ numbers amongst the different floral organ types in response to GA treatment could reflect changes in whorl boundaries early in FM development, or potentially changes in FM starting size.

Under control growth conditions, organ fusion events were detected in sepals and long stamens only and were most common in long stamens (Fig. 6c). GA treatment significantly increased the occurrence of organ fusion in long stamens only ( $p<0.05$; Fig. 6c). Varying degrees of fusion between long stamens were observed under both control growth conditions and under GA treatment (Fig. 6d-f). In contrast, homeosis was found only in short stamens, which always exhibited partial conversion to petal fate (Fig. 6g).
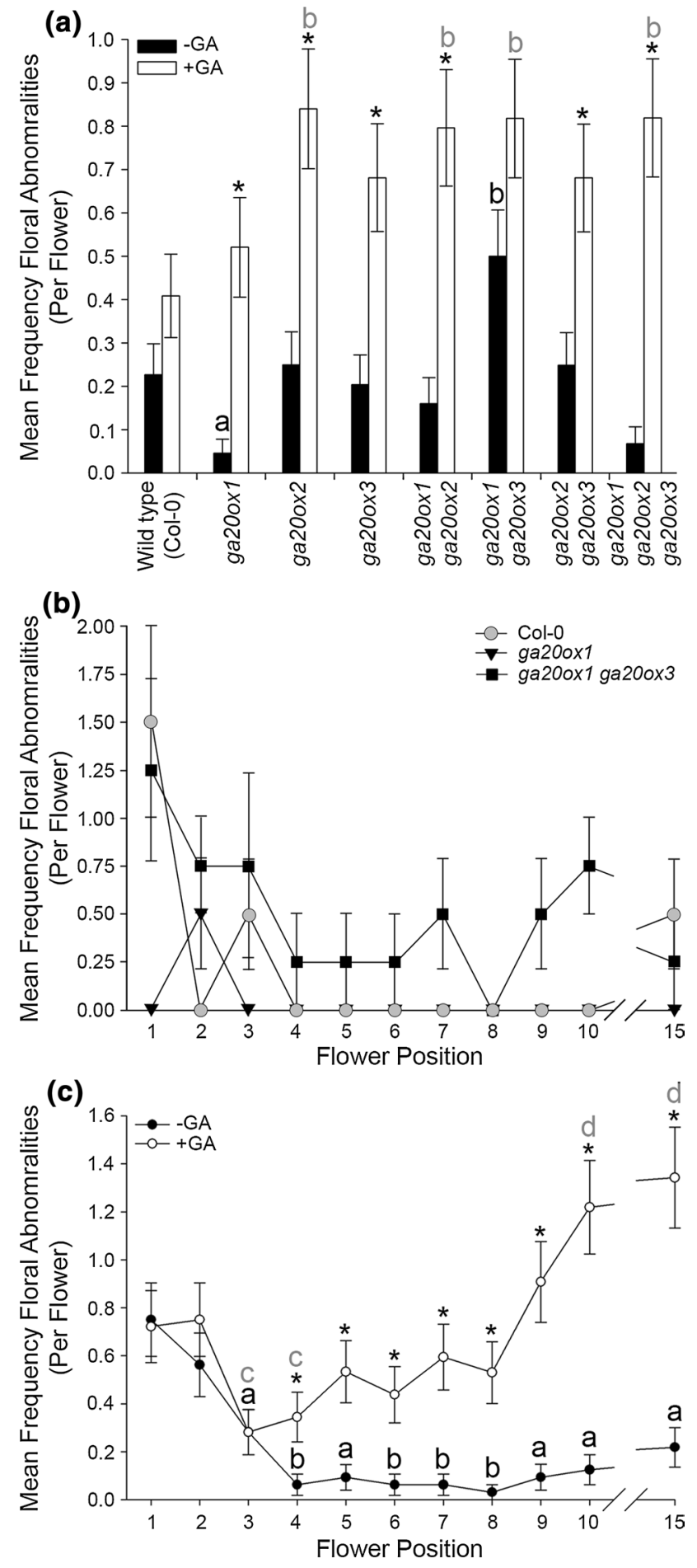

These results indicate that stamens are the floral organ most susceptible to perturbation during early flowering, with all three types of developmental abnormality presenting within whorl 3. The frequency of two of these abnormalities is increased within the early inflorescence under exogenous GA treatment. Whilst there are known regulatory roles for GA during later stamen development (reviewed in Plackett et al. 2011), this is the first phenotypic evidence of a role 
Table 3 Effect of genotype, exogenous GA treatment and flower position on the occurrence of floral abnormalities across the early Arabidopsis inflorescence

\begin{tabular}{|c|c|c|c|c|c|c|c|c|}
\hline \multirow{2}{*}{$\begin{array}{l}\text { Phenotypic } \\
\text { class }\end{array}$} & \multirow{2}{*}{$\begin{array}{l}\text { Level of } \\
\text { analysis }\end{array}$} & \multicolumn{7}{|l|}{ Effects } \\
\hline & & Genotype & GA treatment & Flower position & $\begin{array}{l}\text { Genotype: } \\
\text { GA treat- } \\
\text { ment }\end{array}$ & $\begin{array}{l}\text { Genotype: } \\
\text { flower posi- } \\
\text { tion }\end{array}$ & $\begin{array}{l}\text { GA treatment: } \\
\text { flower position }\end{array}$ & $\begin{array}{l}\text { Genotype: } \\
\text { GA treatment: } \\
\text { flower position }\end{array}$ \\
\hline $\begin{array}{l}\text { All floral abnor- } \\
\text { malities }\end{array}$ & Whole flower & $0.004 * *$ & $<0.001 * * *$ & $<0.001 * * *$ & $0.024 *$ & $\mathbf{0 . 0 3 3}^{*}$ & $<0.001 * * *$ & 0.807 \\
\hline \multirow{5}{*}{$\begin{array}{l}\text { Deviations in } \\
\text { organ number }\end{array}$} & Whole flower & $<0.001 * * *$ & $<0.001 * * *$ & $<0.001 * * *$ & 0.096 & 0.136 & $<0.001 * * *$ & 0.855 \\
\hline & Sepals & 0.360 & $0.009 * *$ & 0.533 & 0.882 & 1.000 & 0.996 & 1.000 \\
\hline & Petals & 0.503 & $0.008 * *$ & 0.822 & 0.836 & 1.000 & 0.995 & 1.000 \\
\hline & Long stamens & 0.128 & $0.036^{*}$ & $0.007 * *$ & 0.279 & 0.903 & 0.118 & 0.999 \\
\hline & Short stamens & $\mathbf{0 . 0 0 6}^{* *}$ & $<0.001 * * *$ & $<0.001 * * *$ & 0.256 & 0.504 & 0.200 & 0.953 \\
\hline \multirow[t]{2}{*}{ Organ fusion } & Whole flower & 0.573 & $0.004 * *$ & 0.242 & 0.751 & 0.940 & 0.843 & 1.000 \\
\hline & Long stamens & 0.377 & $0.002 * *$ & 0.210 & 0.819 & 0.994 & 0.876 & 1.000 \\
\hline Organ homeosis & Short stamens & $0.007 * *$ & 0.378 & 0.608 & 0.817 & 1.000 & 0.963 & 1.000 \\
\hline
\end{tabular}

Summary of $p$ values for main effects (genotype, GA treatment and flower position) and interactions between them (denoted by colons) obtained via GLM (see "Materials and methods"). The floral abnormalities dataset was analysed at a series of hierarchical levels, parsing the data by phenotypic class of abnormality (deviations in organ number, organ fusion, organ homeosis) and the position (whorl) within the flower where the abnormality occurred. Where no abnormalities of a specific type occurred within a particular whorl, that analysis is not shown. Organ homeosis was only observed in short stamens. Statistically significant $p$ values $(p<0.05)$ are highlighted in bold. The numbers of asterisks denotes increasing stringency of statistical significance

$* p<0.05, * * p<0.01, * * * p<0.001$

during earlier floral development when organ number and identity are set. Flower position was found to represent a significant factor in determining the numbers of long and short stamens, which likely account for the position-specific effects identified when analysing all organ types together (cf. Fig. 5c). The differential phenotypic responses between long and short stamens could indicate that long and short stamens might differ in some of the signalling pathways underlying their development.

As described above, when the frequencies of all floral abnormalities were considered collectively, ga20oxl and ga20oxl ga20ox3 showed significant differences from wild type (Fig. 5a), but demonstrated contrary differences (Fig. 5a, b). However, genotype is only a significant factor within short stamens (Table 3), affecting both numbers of short stamens and short stamen homeosis. This argues that the differences between ga20oxl and ga20oxl ga20ox3 centre on short stamen development. In support of this, the effects of genotype on the deviations in numbers of all floral organs (Fig. 7a) can mostly be accounted for through genotype effects on short stamens (Fig. 7b). Within short stamens, a significant difference remained between ga20oxl ga20ox3 and wild type $(p<0.05$; Fig. 7b). Other genotypes also demonstrated significant differences in short stamen number compared to wild type, including ga20ox1 ga20ox2 ga20ox3 $(p<0.05$; Fig. 7b), but interestingly ga20oxl did not $(p>0.05)$. In contrast, the frequency of short stamen homeosis in ga20oxl was significantly reduced compared to the wild type ( $p<0.05$; Fig. $7 \mathrm{c})$, whereas homeosis in ga20oxl ga20ox 3 short stamens was not significantly different from wild type $(p>0.05)$. These results reconcile the apparently contradictory phenotypes of ga20oxl and ga20oxl ga20ox3 found at the level of all floral abnormalities, with the two genotypes affecting separate processes in stamen development.

\section{Discussion}

\section{Floral organ growth is linked within and between flowers of the Arabidopsis inflorescence}

In this work, we demonstrate the presence of significant gradients of floral organ growth in the wild-type Arabidopsis inflorescence: stamen length at flower opening increases with advancing flower position, whilst pistil length remains static. These results indicate that Arabidopsis floral development is not independent of flower position on the early inflorescence and that floral development changes as flowering progresses. This differential growth between stamen and pistil is consistent with an identified trend of increasing probability of silique-set across the early inflorescence. Stamen growth continues after flower opening (Smyth et al. 1990), so changing stamen growth could reflect either 

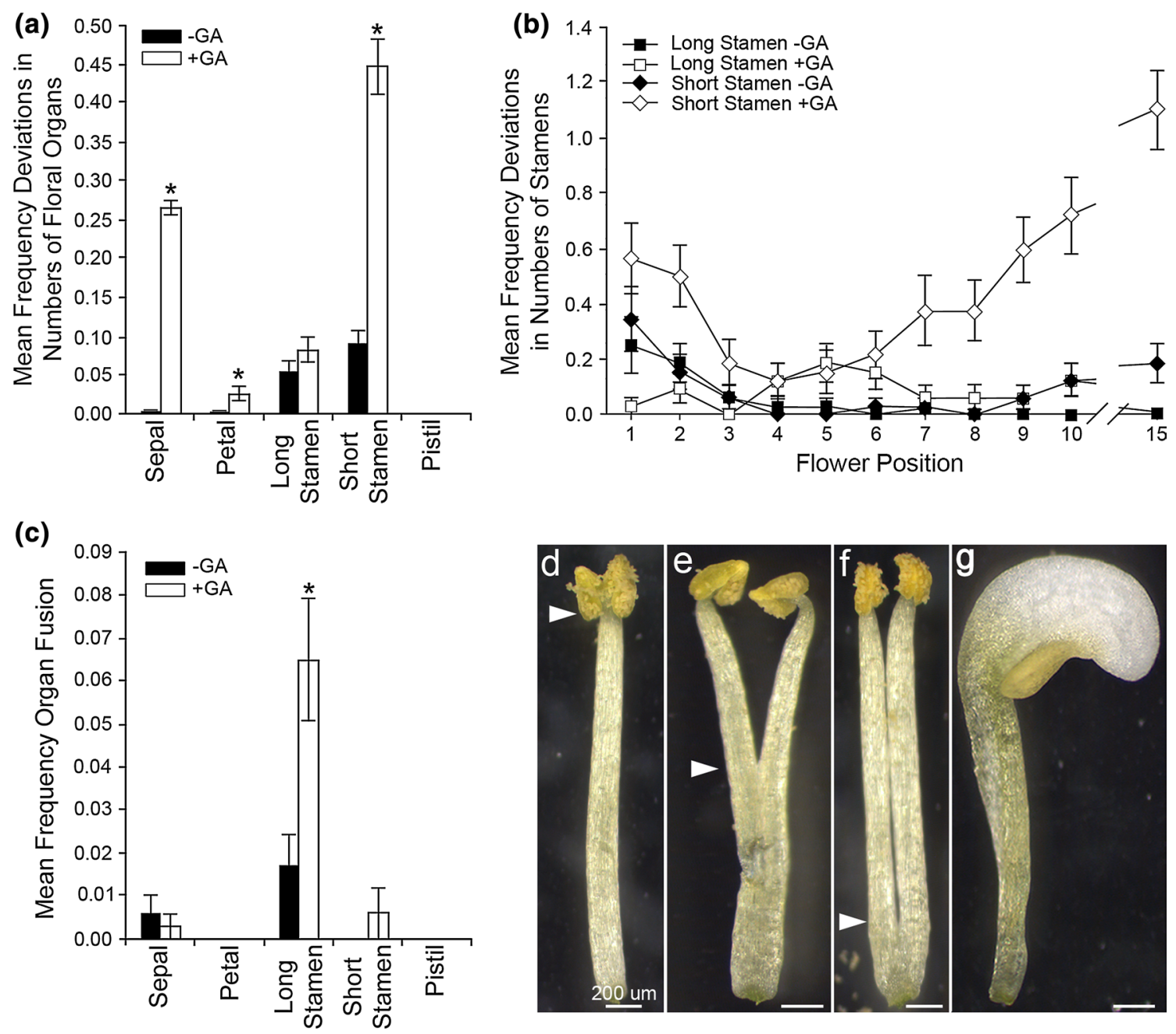

Fig. 6 Exogenous GA treatment most strongly affects floral organ patterning in whorl three (stamens). a Mean frequencies of deviations in floral organ number for each floral organ type under control growth conditions (black) and exogenous GA treatment (white) averaged across all genotypes. Values shown are the mean of 352 independent flowers \pm S.E. Asterisks indicate a significant difference between control and GA-treated conditions $(p<0.05)$. Comparisons were made using LSD values at a significance threshold of 5\% (see Online Resource 5). b Plotted means of the frequencies of deviations in stamen numbers for long and short stamens under control growth conditions (black) and exogenous GA treatment (white) for flower positions $1-10$ and 15 , averaged across all genotypes. Values shown are the mean of 32 independent flowers \pm S.E. At the level of long and short stamens, both GA treatment and flower position have sig-

increased final stamen length in later flowers or accelerated growth in later flowers to achieve the same length earlier in development. Within the same flowers, sepals demonstrated an opposing growth gradient, indicating that these changes are not simply a factor of increasing flower size overall. Floral organs are initiated in concentric whorls, beginning with sepals as the outermost organs and then progressing inwards (Smyth et al. 1990). Thus, changes in size between different organ types could reflect changes during early floral nificant $(p<0.05)$ effects on the frequency of deviations, but there was no significant interaction between the two (long: $p=0.118$, short: $p=0.200$; Table 3). $\mathbf{c}$ Mean frequencies of organ fusion events for each floral organ type under control growth conditions (black) and exogenous GA treatment (white) averaged across all genotypes. Values shown are the mean of 352 independent flowers \pm S.E. Asterisks indicate a significant difference between control and GA-treated conditions $(p<0.05)$. Comparisons were made using LSD values at a significance threshold of 5\% (see Online Resource 5). d-f Examples of organ fusion in stamens; white arrows mark the point of fusion/ splitting between two organs. g Example of stamen homeosis, with conversion of half the organ to petal identity. This phenotype was observed only in short stamens

development, i.e. the timing of organ specification or the size of founder cell populations. The adaptive advantage to the existence of such gradients is as yet unclear, but could represent a mechanism through which plants can improve the chance of successful self-pollination under unfavourable environmental conditions (see below). Alternatively, outcrossing in plant species is usually achieved through differential growth of male and female reproductive organs-for example delaying stamen maturation until the pistil has been 

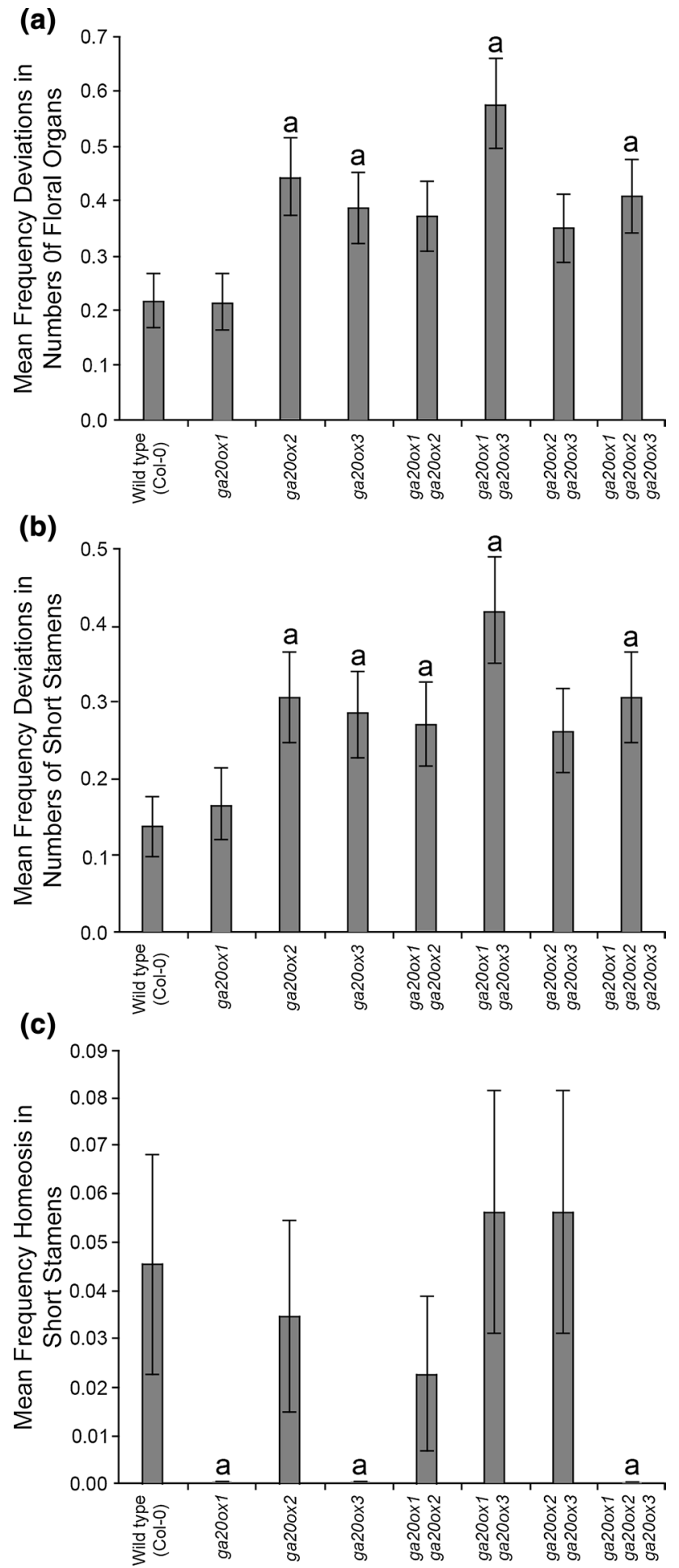

Fig. 7 ga20ox mutants demonstrate differing short stamen phenotypes. Mean frequencies of deviations in floral organ number across the whole flower (a) and within short stamens alone (b), and the mean frequencies of short stamen homeosis (c), by genotype, averaged across growth conditions and all flower positions. Values shown are the mean of 352 independent flowers \pm S.E. Letters denote a significant difference $(p<0.05)$ from wild type. Genotypes marked by different letters are significantly different from one another $(p<0.05)$. Pairwise comparisons were made using LSDs with a significance threshold of 5\% (see Online Resource 5) pollinated (Holtsford and Ellstrand 1992; Sherry and Lord 2000). The gradients found here, during flowering of this typically self-pollinating Arabidopsis laboratory strain, may be related to this mechanism, or act to increase the likelihood of outcrossing in early flowers as part of a bet-hedging strategy to improve reproductive success.

Modelling of floral organ growth across ga20ox mutant inflorescences found that significant gradients in floral organ growth persisted, even in the severely GA-deficient ga20oxl ga20ox 2 ga20ox3. The differences in stamen and pistil growth parameters between ga20ox mutants are sufficient to explain the silique-set phenotypes observed, and we identify potentially non-redundant roles for GA20ox3. The finding that reduced silique-set in ga20oxl is caused by increased pistil growth is surprising, as previous studies have supported expression of GA20oxl within the stamen and not the pistil (Plackett et al. 2012). However, the expression of GA20ox1, GA20ox2 and GA20ox3 has been shown to be interrelated through complex feedback mechanisms (Rieu et al. 2008). GA20ox2 is expressed in pistil tissues during flower development (Plackett et al. 2012) and is significantly up-regulated in ga20oxl plant tissues (Rieu et al. 2008). Thus, feedback up-regulation of GA20ox 2 could feasibly enhance GA biosynthesis in the ga20oxl pistil and promote increased growth. Predictions of GA20ox gene expression changes in mutant backgrounds are complicated further by feedback links to GA3ox (GA biosynthesis) and GA2ox (GA catabolism) gene expression (Yamaguchi 2008). Reduced pistil growth in the ga20ox2 mutant, as identified by linear modelling, is consistent with reported GA20ox2 pistil expression patterns (Plackett et al. 2012), and neither GA20ox1 nor GA20ox3 are up-regulated in ga20ox2 tissues (Rieu et al. 2008). Recent quantification of GA levels from the organs of single wild-type flowers has confirmed the presence of bioactive GA specifically in the pistil, stamen and the receptacle of newly opened flowers, although the flower position sampled on the inflorescence is not indicated (Li et al. 2017).

Both the success of silique-set and floral organ lengths were found to contain inherent variability when comparing between adjacent flower positions along the early inflorescence. In addition, whilst we observed floral phenotypes for early ga20oxl ga20ox 2 flowers similar to those previously reported (Rieu et al. 2008), the observed rates at which spontaneous silique-set recovered appeared to differ between flower position 10 (this study) and approximately flower position 15 (Rieu et al. 2008). Thus, whilst we necessarily refer to differences between specific floral positions based on the results obtained in this study, the positions in themselves are unlikely to hold large biological relevance to the underlying mechanism and instead reflect positions on a gradient that might itself be subject to variability. The most likely cause of the difference between these two studies is 
uncontrolled small variations in experimental growth conditions. Similar phenotypic discrepancies in response to growth conditions have been noted previously in relation to floral development in the GIBBERELLIN INSENSTIVE DWARF 1 (GIDI) GA receptor triple mutant (Iuchi et al. 2007; Griffiths et al. 2007; Willige et al. 2007; Plackett et al. 2014). Environmental factors such as temperature are known to have a significant impact on plant fertility, for example through pollen development and fertilisation (Zinn et al. 2010; De Storme and Geelan 2014). In some plant species, environmental stress results in differential rates of selfpollination and outcrossing through impaired male fertility (Bishop et al. 2017). An interaction between Arabidopsis pollen development, GA and temperature sensitivity has previously been uncovered through the GA signalling negative regulator SPINDLYI (SPYI) (Jacobsen and Olszewski 1993), which is also involved in regulating responses to other abiotic stresses (Qin et al. 2011). Pollen development in the Arabidopsis GAMYB mutant myb33 myb65 also demonstrates increased temperature sensitivity (Millar and Gublar 2005). It would be interesting to determine the extent to which floral organ growth patterns across the inflorescence are influenced by environmental factors.

An important question that remains is what are the pathways through which these gradients are achieved and to what extent they are GA dependent. Significant gradients were found to persist in GA-deficient backgrounds, and gradual recovery of stamen growth in the severely GA-deficient gal-3 mutant has also previously been reported during late flowering (Plackett et al. 2012). As such, these gradients are unlikely to be generated solely through changes in endogenous GA biosynthesis. The presence of bioactive GA is transduced into plant developmental responses through GA-dependent binding of the GID1 receptor protein to growth-repressing DELLA proteins, triggering their rapid degradation (Harberd et al. 2009). There are five DELLA paralogues in Arabidopsis, $G A$ INSENSTIVE (GAI), REPRESSOR OF GAI (RGA) and RGA -LIKE 1 (RGL1), RGL2 and RGL3. These demonstrate differential expression levels in flowers (Tyler et al. 2004), but their precise tissue expression patterns remain unresolved. Arabidopsis mutants lacking or with impaired DELLA function show increased elongation of pistils, stamens and petals compared to wild type (Cheng et al. 2004; Fuentes et al. 2012), demonstrating that under normal development their growth remains under some level of repression. Flowers of the DELLA global mutant (lacking all five DELLA paralogues) in the Arabidopsis Ler ecotype self-pollinate successfully, but have reduced post-pollination fertility (Fuentes et al. 2012). This mutant does demonstrate some reduction in silique-set compared to wild type Ler, but this is not specific to early flowering (Plackett et al. 2014). RGA, RGL1 and RGL2 are the dominant DELLA paralogues regulating floral organ growth in Ler (Cheng et al. 2004). A spontaneous late-flowering recovery of silique-set has been reported in Ler gal-3 mutants lacking two specific combinations of DELLA paralogue, GAI and $R G A$ or $R G A$ and $R G L 2$ (Cheng et al. 2004). The early flowers of rga-24 gai-t6 show impaired floral organ growth similar to gal-3 (Dill and Sun 2001; King et al. 2001), implying that in at least this line the late-stage rescue in silique-set occurs through recovered floral organ growth. Cheng et al. (2004) also found that gal-3 plants lacking GAI, RGA, RGLI and $R G L 2$ produce fertile flowers.

These data, the global mutant phenotype and the reported lack of phenotypic recovery in other pairwise combinations of DELLA paralogue in the gal-3 background (Cheng et al. 2004) suggest that the observed gradients in floral organ growth along the inflorescence do relate directly to the GA signalling pathway, although it would be interesting to test directly for changes in floral organ growth across the inflorescence in non-silique-setting mutants, as silique-set can also be inhibited by reductions in male or female fertility under chemically or genetically GA-overdosed conditions (Jacobsen and Olszewski 1993; Cheng et al. 2004; Fuentes et al. 2012; Plackett et al. 2014). It can also be hypothesised that the mechanisms underlying changing floral organ growth across the inflorescence acts either through modulating GA signal transduction itself or downstream of GA signalling. It would be interesting to determine whether changes in floral organ growth across the inflorescence also occur independently of DELLA-dependent signalling, for example in the gidl triple receptor mutant or the degradation-resistant DELLA mutant gai-1 (Dill et al. 2001).

\section{The recovery of ga200x1 ga200x2 floral organ growth is associated with an implied late-stage developmental checkpoint}

Evidence presented in this study suggests that anther indehiscence in the earliest ga20oxl ga20ox 2 flowers is correlated with a severe reduction of stamen (and petal) growth not seen in flowers with dehiscent anthers at the same flower position. This effect is overlaid onto a gradient of stamen growth similar to that seen in wild type, albeit with a reduced initial stamen length. Anther indehiscence was previously described in early flowers of ga3ox GA biosynthesis mutants (Hu et al. 2008), where stamen development apparently arrested late in flower development (stages 11 or 12). Stamen filament elongation primarily occurs in synchrony with anther maturation during late floral development, and stamen elongation in later ga20ox1 ga20ox2 flowers is reduced at this stage (Plackett et al. 2012). The results of this study therefore support the existence of a GA-dependent, late-stage anther developmental block and further suggest a link, directly or indirectly, with late-stage stamen filament growth. 
Mechanisms linking anther development with filament elongation are currently unknown. The sharp difference in filament lengths between stamens with dehiscent and indehiscent anthers argues against GA itself acting to coordinate the two tissues: in this scenario the relationship between stamen length and GA concentration would be expected to be continuous (analogue). Supporting this is the observation that recent sensitive assays were unable to detect bioactive GA in wild-type petals at flower opening ( $\mathrm{Li}$ et al. 2017). Furthermore, although their average growth was reduced across the early ga20ox1 ga20ox2 inflorescence, growth of pistils and sepals was not affected in flowers with indehiscent anthers. An alternative candidate is the phytohormone jasmonate (JA): the JA biosynthetic mutant opr3 displays arrested anther development and inhibited stamen filament elongation (Stintzi and Browse 2000). However, petal development is apparently unaffected in this mutant. In contrast, the JA biosynthesis mutant defective in anther dehiscence 1 (dadl) demonstrates reduced growth of both stamens and petals during late floral development (Ishiguro et al. 2001). $D A D 1$ expression is restricted to the stamen filament during floral development (Ishiguro et al. 2001), where it is upregulated by GA signalling (Cheng et al. 2009), although treatment with either of these hormones cannot entirely rescue floral phenotypes caused by deficiency of the other (Cheng et al. 2009), suggesting the requirement for other downstream signalling cascades.

How ga20oxl ga20ox2 stamen development spontaneously overcomes this developmental block remains unclear, but presumably relates to changes in signalling along the early inflorescence. Although GA20ox3 has been shown to be up-regulated in the ga20oxl ga20ox 2 pistil and stamen filament (Plackett et al. 2012), Hu et al. (2008) detected no difference in GA levels between early and late ga3ox mutant flowers displaying a similar phenotypic recovery, making it unlikely that ga20oxl ga20ox2 stamen development recovers through direct changes in GA biosynthesis or signalling. Alternatively, pathways downstream of GA signalling might be modulated to promote continued development despite the absence of GA. Our results suggest that any such changes are likely to be located within the developing anther.

\section{Novel roles for GA in floral patterning and stamen development}

Arabidopsis floral development is considered to be a highly determinant, invariant programme of events generating a fixed number of floral organs in sequential, concentric whorls from a floral meristem (FM). Some of the regulatory mechanisms governing these events are already known (reviewed in Airoldi 2010; Irish 2010). However, we detected a significantly higher incidence of abnormal floral development in the first flowers to emerge compared to later flowers across all genotypes. Arabidopsis FMs are produced sequentially from the flanks of the inflorescence meristem (IM), in place of leaf primordia generated during the vegetative phase of development. The positions of lateral organ primordia are determined through auxin maxima in both the vegetative and reproductive phases, the positions of which are influenced by the positions of preceding primordia (Reinhardt et al. 2003; Heisler et al. 2005). Both leaf primordia and FMs are generated in a spiral phyllotaxy, the direction of which is not predetermined and which can change between developing inflorescences on the same plant (Smyth et al. 1990). During the transition of the shoot apical meristem (SAM) to IM identity, the apex increases in size and changes shape (Mishke and Brown 1965). Development in the first FMs may thus be more plastic because they arise without pre-existing primordia of the same type to constrain developmental patterning.

Exogenous GA treatment did not increase the frequency of abnormalities in the first flowers to develop but prolonged their occurrence into later flowering, suggesting that it is necessary to restrict GA signalling to stabilise early floral development. In support of this, we noticed that inflorescence phyllotaxy appeared disturbed in plants grown under GA treatment (Online Resource 4). The GA biosynthetic genes CPS and GA3oxl are both expressed in early FMs prior to the outgrowth of the floral organs (Hu et al. 2008). Until now no developmental functions have been ascribed to GA at this stage of floral development. The FM identity gene $L E A F Y(L F Y)$ has been shown to restrict GA signalling through enhanced GA catabolism to trigger up-regulation of its downstream target APETALAl (API) via DELLA accumulation (Yamaguchi et al. 2014). Our analysis of floral abnormalities at the whole flower level suggested that imbalanced GA signalling across the FM, rather than GA signalling per se, triggers abnormal development. Two genotypes, ga20oxl and ga20oxl ga20ox3, had apparently contradictory effects on the frequency of abnormalities as a result of influencing different developmental processes (short stamen homeosis and short stamen numbers, respectively). The expression patterns of the GA20ox paralogues have not been mapped within the early FM, but could cause localised differences in bioactive GA levels through regulating local availability of the GA3ox substrate $\mathrm{GA}_{9}$.

We identified significant deviations in the numbers of floral organs produced under GA treatment. Changes to the number of organs that develop might reflect alterations to the relative sizes of the concentric whorls specified within the FM, either directly regulated by GA signalling or indirectly through a change in initial FM size: a larger FM could allow more sepals to arise at the periphery (as was found), the altered geometry subsequently affecting patterning in the later-developing inner whorls. The tendency towards loss of short stamens can also be explained by such a mechanism, as 
an outcome of their late emergence after the establishment of long stamens (Smyth et al. 1990). However, we did not find any clear correlation between changes in the number of organs across different whorls. Organ fusion events indicate defects in organogenesis beyond organ specification. Separate floral organ primordia are established within each whorl by the creation of boundaries between adjacent organs, comprised of specialised cell types (reviewed in Aida and Tasaka 2006; Wang et al. 2016). A number of transcription factors regulate stamen boundary formation, including $C U P$ SHAPED COTYLEDON1 (CUC1) and CUC2 (Aida et al. 1997), FUSED FLORAL ORGANS1 (FFO1), FFO2 and FFO3 (Levin et al. 1998), UNUSUAL FLORAL ORGANS (UFO; Laufs et al. 2003) and HANABA TARANU (HAN; Zhao et al. 2004). Organ loss and/or stamen fusion has been observed in a number of mutants for these genes, and auxin signalling has been implicated as an organising factor (Furutani et al. 2004). The changes in organ number and organ fusions observed in this study could arise through mis-regulation of these or other boundary genes in very early flowers and under exogenous GA treatment. Regulation by GA might be direct, or changes in size of the initial FM or whorl domain might result in indirect perturbations downstream.

Abnormalities in floral organ development occurred most frequently in stamens. Similar stamen defects arise when auxin biosynthesis or polar auxin transport are disrupted (Cardarelli and Cecchetti 2014). Later stages of stamen development are heavily regulated by GA signalling (reviewed in Plackett et al. 2011). Floral organ identity is specified through interactions of MADS-box genes following the ABCE model (reviewed in Irish 2010): stamens are determined by interaction between the B class MADS-box genes PISTILLATA (PI) and APETALA3 (AP3) and the C class gene AGAMOUS (AG). AP3, $P I$ and $A G$ are all known targets of GA signalling, either directly or via the GA downstream target $L F Y$ (Blázquez et al. 1998; Wagner et al. 1999; Yu et al. 2004). Prolonged $A G$ expression during floral development is required to maintain stamen identity (Bowman et al. 1991; Ito et al. 2007), and as well as being a downstream target of GA signalling, AG up-regulates GA3ox 1 (Gómez-Mena et al. 2005). Stamens are converted to petals in ag loss-of-function mutants (Coen and Meyerowitz 1991) and GA treatment can also promote petal development in ap1 and ap2 homeotic mutant backgrounds (Okamuro et al. 1997), where petals are converted to stamen identity. The homeotic short stamen phenotype observed here is presumably linked to GA-dependent changes in $\mathrm{A}, \mathrm{B}$ or $\mathrm{C}$ gene expression, although mis-regulation of some boundary genes such as $U F O$ can also lead to the formation of mosaic floral organ identities (Levin and Meyerowitz 1995).

The analysis of Arabidopsis early flowering presented here has thus identified new potential roles for GA signalling during the very early stages of floral development. The developmental plasticity demonstrated by the first flowers represents an excellent vehicle to better understand the regulation of these early development processes.

Author contribution statement ALP, PH, ST and ZAW designed research. ARGP conducted experimental work. SJP conducted modelling and statistical analysis. All authors read and approved the manuscript.

Acknowledgements This work was supported by a Rothamsted quota studentship to ARGP and by grants P16508 and BB/J001295/1, funded by the Biotechnology and Biological Sciences Research Council of the UK, which also provides support to Rothamsted Research through funding of the 20:20 Wheat ${ }^{\odot}$ and Designing Future Wheat Integrated Strategic Programmes (ALP, PH, SJP and ST). We are grateful to I. Pearman, A. Griffin and other glasshouse staff for plant husbandry.

\section{Compliance with ethical standards}

Conflict of interest The authors declare that they have no conflict of interest.

Open Access This article is distributed under the terms of the Creative Commons Attribution 4.0 International License (http://creativecommons.org/licenses/by/4.0/), which permits unrestricted use, distribution, and reproduction in any medium, provided you give appropriate credit to the original author(s) and the source, provide a link to the Creative Commons license, and indicate if changes were made.

\section{References}

Aida M, Tasaka M (2006) Morphogenesis and patterning at the organ boundaries in the higher plant shoot apex. Plant Mol Biol 60:915-928

Aida M, Ishida T, Fukaki H, Fujisawa H, Tasaka M (1997) Genes involved in organ separation in Arabidopsis: an analysis of the cup-shaped cotyledon mutant. Plant Cell 9:841-857

Airoldi CA (2010) Determination of sexual organ development. Sex Plant Reprod 23:53-62

Bishop J, Jones HE, O'Sullivan DM, Potts SG (2017) Elevated temperature drives a shift from selfing to outcrossing in the insect-pollinated legume, faba bean (Vicia faba). J Exp Bot 68:2055-2063

Blázquez MA, Green R, Nilsson O, Sussman MR, Weigel D (1998) Gibberellins promote flowering of Arabidopsis by activating the $L E A F Y$ promoter. Plant Cell 10:791-800

Bowman JA, Drews GN, Meyerowitz EM (1991) Expression of the Arabidopsis floral homeotic gene AGAMOUS is restricted to specific cell types late in flower development. Plant Cell 3:749-758

Cardarelli M, Cecchetti V (2014) Auxin polar transport in stamen formation and development: how many actors? Front Plant Sci 5:333

Cheng H, Qin L, Lee S, Fu X, Richards DE, Cao D, Luo D, Harberd NP, Peng J (2004) Gibberellin regulates floral development via suppression of DELLA protein function. Development 131:1055-1064

Cheng H, Song S, Xiao L, Soo HM, Cheng Z, Xie D, Peng J (2009) Gibberellin acts through jasmonate to control the expression of 
MYB21, MYB24 and MYB57 to promote stamen filament growth in Arabidopsis. PLoS Genet 5:e1000440

Chiang H-H, Hwang I, Goodman HM (1995) Isolation of the Arabidopsis GA4 locus. Plant Cell 7:195-201

Coen ES, Meyerowitz EM (1991) The war of the whorls: genetic interactions controlling flower development. Nature 353:31-37

Coles JP, Phillips AL, Croker SJ, García-Lepe L, Lewis MJ, Hedden P (1999) Modification of gibberellin production and plant development in Arabidopsis by sense and antisense expression of gibberellin 20-oxidase genes. Plant J 17:547-556

De Storme N, Geelan D (2014) The impact of environmental stress on male reproductive development in plants: biological processes and molecular mechanisms. Plant, Cell Environ 37:1-18

Dill A, Sun TP (2001) Synergistic derepression of gibberellin signalling by removing RGA and GAI function in Arabidopsis thaliana. Genetics 159:777-785

Dill A, Jung SH, Sun TP (2001) The DELLA motif is essential for gibberellin-induced degradation of RGA. Proc Natl Acad Sci USA 98:14162-14167

Fuentes S, Ljung K, Sorefan K, Alvey E, Harberd NP, Østergaard L (2012) Fruit growth in Arabidopsis occurs via DELLA-dependent and DELLA-independent gibberellin responses. Plant Cell 24:3982-3996

Furutani M, Vernoux T, Traas J, Kato T, Tasaka M, Aida M (2004) PIN-FORMEDI and PINOID regulate boundary formation and cotyledon development in Arabidopsis embryogenesis. Development 131:5021-5030

Gomez KA, Gomez AA (1984) Statistical procedures for agricultural research. Wiley, New York

Gómez-Mena C, de Folter S, Costa MMR, Angenent GC, Sablowski $\mathrm{R}$ (2005) Transcriptional program controlled by the floral homeotic gene AGAMOUS during early organogenesis. Development 132:429-438

Griffiths J, Murase K, Rieu I, Zentella R, Zhang ZL, Powers SJ, Gong F, Phillips AL, Hedden P, Sun TP, Thomas SG (2007) Genetic characterization and functional analysis of the GID1 gibberellin receptors in Arabidopsis. Plant Cell 18:3399-3414

Harberd N, Belfield E, Yasamura Y (2009) The angiosperm gibberellin-GID1-DELLA growth regulatory mechanism: how an "inhibitor of an inhibitor" enables flexible response to fluctuating environments. Plant Cell 21:1328-1339

Heisler MG, Ohno C, Das P, Sieber P, Reddy GV, Long JA, Meyerowitz EM (2005) Patterns of auxin transport and gene expression during primordium development revealed by live imaging of the Arabidopsis inflorescence meristem. Curr Biol 15:1434-1448

Holtsford TP, Ellstrand NC (1992) Genetic and environmental variation in floral traits affecting outcrossing rate in Clarkia tembloriensis (Onagraceae). Evolution 46:216-225

Hu J, Mitchum MG, Barnaby N et al (2008) Potential sites of bioactive gibberellin production during reproductive growth in Arabidopsis. Plant Cell 20:320-336

Huang S, Raman AS, Ream JE, Fujiwara H, Cerny RE, Brown SM (1998) Overexpression of 20-oxidase confers a gibberellin-overproduction phenotype in Arabidopsis. Plant Physiol 118:773-781

Irish VF (2010) The flowering of Arabidopsis flower development. Plant J 61:1014-1028

Ishiguro S, Kawai-Oda A, Ueda J, Nishida I, Okada K (2001) The DEFECTIVE IN ANTHER DEHISCENCE1 gene encodes a novel phospholipase A1 catalyzing the initial step of jasmoinc acid biosynthesis, which synchronises pollen maturation, anther dehiscence, and flower opening in Arabidopsis. Plant Cell 13:2191-2209

Ito T, Ng KH, Lim TZ, Yu H, Meyerowitz EM (2007) The homeotic protein AGAMOUS controls late stamen development by regulating a jasmonate biosynthetic gene in Arabidopsis. Plant Cell 19:3516-3529
Itoh H, Tanaka-Ueguchi M, Kawaide H, Chen X, Kamiya Y, Matsuoka M (1999) The gene encoding tobacco gibberellin 3 $\beta$-hydroxylase is expressed at the site of GA action during stem elongation and flower organ development. Plant J 20:15-24

Iuchi S, Susuki H, Kim YC, Iuchi A, Kuromori T, Ueguchi-Tanaka M, Asami T, Yamaguchi I, Matsuoka M, Kobayashi M, Nakajima M (2007) Multiple loss-of-function of Arabidopsis gibberellin receptor AtGID1s completely shuts down a gibberellin signal. Plant J 50:958-966

Jacobsen SE, Olszewski NE (1993) Mutations in the SPINDLY locus of Arabidopsis alter gibberellin signal transduction. Plant Cell 5:887-896

King KE, Moritz T, Harberd NP (2001) Gibberellins are not required for normal stem growth in Arabidopsis thaliana in the absence of GAI and RGA. Genetics 159:767-776

Koornneef M, Van der Veen JH (1980) Induction and analysis of gibberellin sensitive mutants in Arabidopsis thaliana (L) heyhn. Theor Appl Genet 58:257-263

Laufs P, Coen E, Kronenberger J, Traas J, Doonan J (2003) Separable roles of $U F O$ during floral development revealed by conditional restoration of gene function. Development 130:785-796

Levin JZ, Meyerowitz EM (1995) UFO: An Arabidopsis gene involved in both floral meristem and floral organ development. Plant Cell 7:529-548

Levin JZ, Fletcher JC, Chen X, Meyerowitz EM (1998) A genetic screen for modifiers of $U F O$ meristem activity identifies three novel FUSED FLORAL ORGANS genes required for early flower development in Arabidopsis. Genetics 149:579-595

Li D, Guo Z, Liu C, Li J, Xu W, Chen Y (2017) Quantification of near-attomole gibberellins in floral organs dissected from a single Arabidopsis flower. Plant J 91:547-557

McCullagh P, Nelder JA (1989) Generalized linear models. Chapman and Hall, London

Millar AA, Gublar F (2005) The Arabidopsis GAMYB-like genes, $M Y B 33$ and $M Y B 65$, are microRNA-regulated genes that redundantly facilitate anther development. Plant Cell 17:705-721

Mishke JP, Brown JAM (1965) Development of vegetative and floral meristems of Arabidopsis thaliana. Am J Bot 52:533-537

Mitchum MG, Yamaguchi S, Hanada A, Kuwahara A, Yoshioka Y, Kato T, Tabata S, Kamiya Y, Sun TP (2006) Distinct and overlapping roles of two gibberellin 3-oxidases in Arabidopsis development. Plant J 45:804-818

Okamuro JK, Szeto W, Prass-Lotys C, Jofuku KD (1997) Photo and hormonal control of meristem identity in the Arabidopsis flower mutants apetala2 and apetala1. Plant Cell 9:37-47

Otsuka M, Kenmoku H, Ogawa M, Okada K, Mitsuhashi W, Sassa T, Kamiya Y, Toyomasu T, Yamaguchi S (2004) Emission of entkaurene, a diterpenoid hydrocarbon precursor for gibberellins, into the headspace from plants. Plant Cell Physiol 45:1129-1139

Plackett ARG, Thomas SG, Wilson ZA, Hedden P (2011) Gibberellin control of stamen development: a fertile field. Trends Plant Sci 16:568-578

Plackett ARG, Powers SJ, Fernandez-Garcia N et al (2012) Analysis of the developmental roles of the Arabidopsis gibberellin 20-oxidases demonstrates that GA20oxl, -2 and -3 are the dominant paralogues. Plant Cell 24:941-960

Plackett ARG, Ferguson AC, Powers SJ, Wanchoo-Kohli A, Phillips AL, Wilson ZA, Hedden P, Thomas SG (2014) DELLA activity is required for successful pollen development in the Columbia ecotype of Arabidopsis. New Phytol 201:825-836

Prusinkiewicz P, Erasmus Y, Lane B, Harder LD, Coen E (2007) Evolution and development of inflorescence architectures. Science 316:1452-1456

Qin F, Kodaira KS, Maruyama K, Mizoi J, Tran LSP, Fujita Y, Morimoto K, Shinozaki K, Yamaguchi-Shinozaki K (2011) SPINDLY, 
a negative regulator of gibberellic acid signalling, is involved in the plant abiotic stress response. Plant Physiol 157:1900-1913

Regnault T, Davière J-M, Wild M, Sakvarelidze-Achard L, Heintz D, Carrera Bergua E, Lopez Diaz I, Gong G, Hedden P, Achard P (2015) The gibberellin precursor $\mathrm{GA}_{12}$ acts as a long-distance growth signal in Arabidopsis. Nat Plants 1:15073. https://doi. org/10.1038/nplants.2015.73

Reinhardt D, Pesce ER, Stieger P, Mendel T, Baltensberger K, Bennett M, Traas J, Friml J, Kuhlemeier C (2003) Regulation of phyllotaxis by polar auxin transport. Nature $426: 255-260$

Rieu I, Ruiz-Rivero O, Fernandez-Garcia N et al (2008) The gibberellin biosynthetic genes AtGA20ox1 and AtGA20ox2 act, partially redundantly, to promote growth and development throughout the Arabidopsis lifecycle. Plant J 53:488-504

Sherry RA, Lord EM (2000) A comparative developmental study of the selfing and outcrossing flowers of Clarkia tembloriensis (Onagraceae). Int J Plant Sci 161:563-574

Silverstone AL, Chang C-W, Krol E, Sun T-P (1997) Developmental regulation of the gibberellin biosynthetic gene GAl in Arabidopsis thaliana. Plant J 12:9-19

Silverstone AL, Jung HS, Dill A, Kawaide H, Kamiya Y, Sun TP (2001) Repressing a repressor: gibberell-ininduced rapid reduction of the RGA protein in Arabidopsis. Plant Cell 13:1555-1565

Smyth DR, Bowman JL, Meyerowitz EM (1990) Early flower development in Arabidopsis. Plant Cell 2:755-767

Stintzi A, Browse J (2000) The Arabidopsis male-sterile mutant, opr3, lacks the 12-oxophytodienoic acid reductase required for jasmonate synthesis. Proc Natl Acad Sci USA 97:10625-10630

Tyler L, Thomas SG, Hu J, Dill A, Alonso JM, Ecker JR, Sun TP (2004) DELLA proteins and gibberellin-regulated seed germination and floral development in Arabidopsis. Plant Physiol 135:1008-1019
Vivian-Smith A, Koltunow AM (1999) Genetic analysis of growthregulator-induced parthenocarpy in Arabidopsis. Plant Physiol 121:437-451

Wagner D, Sablowski RWM, Meyerowitz EM (1999) Transcriptional activation of APETALA1 by LEAFY. Science 285:582-584

Wang Q, Hasson A, Rossmann S, Theres K (2016) Divide et impera: boundaries shape the plant body and initiate new meristems. New Phytol 209:485-498

Willige BC, Ghosh S, Nill C, Zourelidou M, Dohmann EMN, Maier A, Schwechheimer C (2007) The DELLA domain of GA INSENSITIVE mediates the interaction with the GA INSENSITIVE DWARF1A gibberellin receptor of Arabidopsis. Plant Cell 19:1209-1220

Yamaguchi S (2008) Gibberellin metabolism and its regulation. Ann Rev Plant Biol 59:225-251

Yamaguchi N, Winter CM, Wu MF, Kanno Y, Yamaguchi A, Seo M, Wagner D (2014) Gibberellin acts positively then negatively to control the onset of flower formation in Arabidopsis. Science 344:638-641

Yu H, Ito T, Zhao Y, Peng J, Kumar P, Meyerowitz EM (2004) Floral homeotic genes are targets of gibberellin signalling in flower development. Proc Natl Acad Sci USA 101:7827-7832

Zhao Y, Medrano L, Ohashi K, Fletcher JC, Yu H, Sakai H, Meyerowitz EM (2004) HANABA TARANU is a GATA transcription factor that regulates shoot apical meristem and flower development in Arabidopsis. Plant Cell 16:2586-2600

Zinn KE, Tunc-Ozdemier M, Harper JF (2010) Temperature stress and plant sexual reproduction: uncovering the weakest links. J Exp Bot 61:1959-1968 